\title{
Nonlinear Vibration of a Nonlocal Functionally Graded Beam on Fractional Visco-Pasternak Foundation
}

Nikola Nešić ( $\square$ nikola.nesic@pr.ac.rs )

Univerzitet u Pristini https://orcid.org/0000-0001-6237-4735

\section{Milan Cajić}

Swansea University College of Engineering

\section{Danilo Karličić}

Mathematical Institute of the Serbian Academy of Sciences and Arts: Matematicki institut Srpske akademije nauka i umetnosti

\section{Aleksandar Obradovic}

University of Belgrade Faculty of Mechanical Engineering: Univerziteta u Beogradu Fakultet Masinski

\section{Julijana Simonović}

University of Niš: Univerzitet u Nisu

\section{Research Article}

Keywords: Nonlocal strain gradient theory, Functionally graded beams, Fractional Pasternak layer, Duffing oscillator, Fractional damping, Incremental harmonic balance method

Posted Date: September 21st, 2021

DOl: https://doi.org/10.21203/rs.3.rs-231211/v1

License: (c) (i) This work is licensed under a Creative Commons Attribution 4.0 International License. Read Full License

Version of Record: A version of this preprint was published at Nonlinear Dynamics on January 17th, 2022. See the published version at https://doi.org/10.1007/s11071-021-07081-z. 


\title{
Nonlinear vibration of a nonlocal functionally graded beam on fractional visco-Pasternak foundation
}

\author{
Nikola Nešić · Milan Cajić · Danilo Karličić · Aleksandar Obradović · Julijana \\ Simonović
}

Received: date / Accepted: date

\begin{abstract}
This paper investigates the nonlinear dynamic behavior of a nonlocal functionally graded Euler-Bernoulli beam resting on a fractional visco-Pasternak foundation and subjected to harmonic loads. The proposed model captures both, nonlocal parameter considering the elastic stress gradient field and a material length scale parameter considering the strain gradient stress field. Additionally, the von Karman strain-displacement relation is used to describe the nonlinear geometrical beam behavior. The power-law model is utilized to represent the material variations across the thickness direction of the functionally graded beam. The following steps are conducted in this research study. At first, the governing equation of motion is derived using Hamilton's principle and then reduced to the nonlinear fractional order differential equation through the single-mode Galerkin approximation. The methodology to determine steady-state amplitude-
\end{abstract}

Nikola Nešić

Faculty of Technical Sciences, University of Priština, Kosovska Mitrovica, Serbia

E-mail: nikola.nesic@pr.ac.rs

Milan Cajić

College of Engineering, Swansea University, United Kingdom (present address)

Mathematical institute of the Serbian Academy of Sciences and Arts,

Kneza Mihaila 36, Belgrade, Serbia

E-mail: mcajic@mi.sanu.ac.rs

Danilo Karličić

Mathematical institute of the Serbian Academy of Sciences and Arts,

Kneza Mihaila 36, Belgrade, Serbia

E-mail: danilok@mi.sanu.ac.rs

Aleksandar Obradović

Faculty of Mechanical Engineering, University of Belgrade, Belgrade, Serbia

E-mail: aobradovic@mas.bg.ac.rs

Julijana Simonović

Faculty of Mechanical Engineering, University of Niš, Niš, Serbia

E-mail: julijana.simonovic@masfak.ni.ac.rs frequency responses via incremental harmonic balance method and continuation technique is presented. The obtained periodic solutions are verified against the perturbation multiple scales method for the weakly nonlinear case and numerical integration Newmark method in the case of strong nonlinearity. It has been shown that the application of the incremental harmonic balance method in the analysis of nonlocal strain gradient theory-based structures, can lead to more reliable studies for strongly nonlinear systems. In the parametric study is shown that, on one hand, parameters of the viscoPasternak foundation and power-law index remarkable affect the response amplitudes. On the contrary, the nonlocal and the length scale parameters are having a small influence on the amplitude-frequency response. Finally, the effects of the fractional derivative order on the system's damping are displayed at time response diagrams and subsequently discussed.

Keywords Nonlocal strain gradient theory $\cdot$ Functionally graded beams · Fractional Pasternak layer · Duffing oscillator · Fractional damping · Incremental harmonic balance method

\section{Introduction}

Structures with physical properties which are varied continuously and gradually along a certain direction are known as functionally graded (FG) materials $[17,38,60]$. The major advantage of such materials that are emphasized in the engineering practice is that they lack stress concentration, which is a common problem at interfaces of conventional laminated composites $[17,38]$. FG materials are usually composed of two different material phases such as metal and ceramics. Despite a significant amount of work done in the field of FG structures, there is still a lot of space and need for the investigation of MEMS/NEMS systems based on FG 
materials. Nanobeams and nanoplates are utilized in different MEMS/NEMS devices [48, 35], such as microactuators [44, 20], microswitches [70], micro sensors [42], nanoscale resonators [19], energy harvesting nanodevices [65], etc. For studying the dynamic behavior of such systems various approaches based on experiments, molecular dynamics simulations, and continuum mechanics are already employed in the literature [8, 14, 41, 28, 39, 52]. However, it can be time and skill-demanding to set up and validate the experiment or to implement molecular dynamics simulations for such structures. For these reasons, size-dependent continuum mechanics models gain popularity due to their simplicity in predicting the mechanical behavior of micro/nano-scaled structural systems. Various non-classical elasticity theories are used to capture the size effects in micro and nanostructures. Among them, the most vastly used continuum theories for studying the nanostructures are: nonlocal elasticity theory [52, 50, 47], strain gradient theory [28], modified couple stress theory (or modified strain gradient theory) [67], and the surface elasticity theory [62]. Some experiments [28] revealed that nonlocal elasticity theory shows limitations in displaying the stiffness-hardening effect. This deficiency can be avoided when nonlocal strain gradient theory (NLSGT) is used as originally described by Lim et al. [36], which includes both nonlocal and length scale effects into the consideration.

Many studies employed NLSGT when analyzing the mechanical behavior of FG structures. Gao et al. [15] investigated the nonlinear free vibration of FG circular nanotubes using NLSGT and two steps perturbation method. Janevski [23, 24] studied linear vibration, stability, and buckling of nonlocal strain gradient Euler-Bernoulli and Timoshenko beams under the influence of temperature. El-Borgi et al. [8] investigated the free and forced vibration response of a simply supported FG beam resting on the nonlinear elastic foundation. The authors applied the perturbation method of multiple scales to obtain the amplitude-frequency curves of the system. Other authors [21] studied the heat-induced nonlinear vibration of FG capacitive nanobeam within the framework of NLSGT. The semi-analytic perturbation method of averaging was applied to obtain the governing equations and study the steady-state responses. They also used a shooting technique in conjunction with the Floquet theory for capturing the periodic motions and examining their stability. Wang and Shen [63] investigated the lateral nonlinear vibration of an axially moving simply supported viscoelastic nanobeam based on NLSGT. They used a direct multiscale method to obtain the steady-state amplitude-frequency response in the subharmonic parametric resonance state as well as the Routh-Hurwitz criterion to determine the stability of the (non-) zero equilibrium solution. Jalaei et al. [22] investigated the dynamic stability of a temperature-dependent Timoshenko functionally graded nanobeam exposed to the axial excitation load and magnetic field in a thermal environment. The authors used Navier's and Bolotin's methodbased approach to solve the problem. Li et al. [32] studied the longitudinal vibration of rods also using the NLSGT and derived analytical solutions for predicting the natural frequencies and mode shapes for specified boundary conditions. They discovered that the NLSGT rod model exerts a stiffness-softening effect when the nonlocal parameter is larger than the length scale parameter and exerts a stiffnesshardening effect in the opposite case. Li [34] investigated the vibration of axially FG beams based on NLSGT and EulerBernoulli beam theory and solved the problem via the generalized differential quadrature method. Simsek [56] proposed a beam model for nonlinear free vibration of an FG nanobeam with immovable ends based on the NLSGT and Euler-Bernoulli (EB) beam theory in conjunction with the von-Karman's geometric nonlinearity. Liu [37] examined the nonlinear vibrational behavior of FG sandwich NLSGT nanobeams in the presence of initial geometric imperfection. Nonlinearity induced by the von Karman theory and a cosine function similar to the mode shape form is employed to describe the geometric imperfection mode. They used He's variational principle to solve a nonlinear differential equation and obtain nonlinear frequency. Based on NLSGT, Li and $\mathrm{Hu}$ [31] and Zhen and Zhou [73] studied the wave propagation in fluid-conveying viscoelastic single-walled carbon nanotubes. Moreover, Li [33] investigated the fluid critical flow velocities of fluid-conveying microtubes modeled using NLSGT and Timoshenko and Euler-Bernoulli beam theories.

Zhang et al. [71] studied the nonlinear dynamic response of a simply supported fractional viscoelastic beam subjected to transverse harmonic excitation. By using the averaging method, the authors obtained a steady-state response of a single-mode system. Numerical results are determined by an algorithm based on the fractional-order Grünwald-Letnikov derivative and verified with analytical results. Eyebe [13] investigated the nonlinear vibration of a nanobeam resting on a fractional-order Winkler-Pasternak foundation by using the D'Alembert principle to obtain the governing equations and a method of multiple scales to approximate the resulting nonlinear problem. Further, Lewandowski [29] investigated the nonlinear, steady-state vibration and stability of harmonically excited fractional viscoelastic beams. The viscoelastic material of the beams is described by using the Zener rheological model with fractional derivatives. Amplitude equations are obtained by using the finite element and the harmonic balance method in conjunction with the continuation method.

In the paper [5], a homogeneous Euler Bernoulli beam on a Winkler-type nonlinear, viscoelastic, and unilateral or bilateral foundation was considered. The presented model was subjected to multiple concentrated or distributed trans- 
verse static or dynamic loads. The IHB method was suited for obtaining the nonlinear frequency response of the system. Obtained and presented amplitude-frequency diagrams were expectantly similar to diagrams from our study. However, the considered parameters set were different both regarding the loading and the foundation properties. Their interest was the reaction of the foundation, which models foam materials, both to compression and tension. Instead, we research the influence of the parameters of the fractional-order model of foundation that can represent a range of different materials from foams to rubbers. Furthermore, our numerical calculations were verified with two other numerical methods, namely with multiple time scales and Newmark methods.

In this work, a detailed investigation of the nonlinear vibration of the nonlocal strain gradient Euler-Bernoulli beam resting on the fractional visco-Pasternak foundation and subjected to harmonic loads is performed. The suggested model contains both, nonlocal parameter considering the nonlocal elastic stress field and a material length scale parameter considering the strain gradient stress field. The following steps are conducted in this research study. First, the governing equation of motion is derived using Hamilton's principle and then reduced to the nonlinear fractional order differential equation via Galerkin approximation. The methodology to determine steady-state amplitude-frequency responses via incremental harmonic balance method and continuation technique is presented. The obtained periodic solutions are verified against the perturbation multiple scales method and numerical integration Newmark method. At last, a detailed parametric study is performed to show the influence of powerlaw index, nonlocal parameter, length scale parameter, parameters of fractional visco-Pasternak foundation, and load factors on the amplitude-frequency response curves of the proposed nonlinear problem. Additionally, the effects of the fractional derivative order and power-law index on the system's damping are displayed at time response diagrams and subsequently discussed.

\section{Preliminaries}

\subsection{Fractional derivative}

The vibration of the deformable structures grounded on the different types of the foundation is present in a wide range of practical structures. Usually, the impact of the foundation layer has great importance and has to be modeled appropriately. The model of the foundation with different properties can be found in the literature [69]. Visco-Pasternak foundation model used in our study was upgraded with fractionalorder time derivatives of the deformation function. This allows us to encompass the whole range of grounds with different properties. We will use the Riemann-Liouville defini- tion (Eq.(1)) when considering the IHB and multiple scales solutions and the Grünwald-Letnikov definition (Eq.(2)) in the case of the Newmark method $[49,58]$. The RiemannLiouville definition is equal to Grünwald-Letnikov definition [49], and these two definitions are equivalent for a wide class of functions and are often used in real physical and engineering problems. For this reason, we can use one definition and then turn to another when calculating the frequency responses by approximate and numerical methods. Here, both definitions are given for clarity. The RiemannLiouville fractional time derivative can be defined as left or right, and we will use the left one.

The left Riemann-Liouville derivative of the continuous and differentiable, on a time interval $[\mathrm{a}, \mathrm{b}]$, function $f(t)$, is defined as:

${ }_{a} D_{t}^{\alpha} f(t)=\frac{1}{\Gamma(n-\alpha)} \frac{d^{n}}{d t^{n}} \int_{a}^{t} \frac{f(\tau)}{(t-\tau)^{\alpha-n+1}} d \tau, \quad t \in[a, b]$,

where $\alpha$ is the fractional-order derivative parameter and $n \in$ $N$ is a natural number such that $n-1<\alpha<n$. To preserve the physicality of the structure, we limit our investigation to the case when fractional derivative order is $\alpha \in[0,1)$.

Grünwald-Letnikov definition of a fractional derivative is given as

${ }_{a} D_{t}^{\alpha} f(t)=\lim _{h \rightarrow 0} \frac{{ }_{a} \Delta_{t}^{\alpha} f(t)}{h^{\alpha}}$,

where

${ }_{a} \Delta_{t}^{\alpha} f(t)=\sum_{j=0}^{\left[\frac{t-a}{h}\right]}(-1)^{j}\left(\begin{array}{c}\alpha \\ j\end{array}\right) f(t-j h)$,

and $[x]$ means the integer part of $x$.

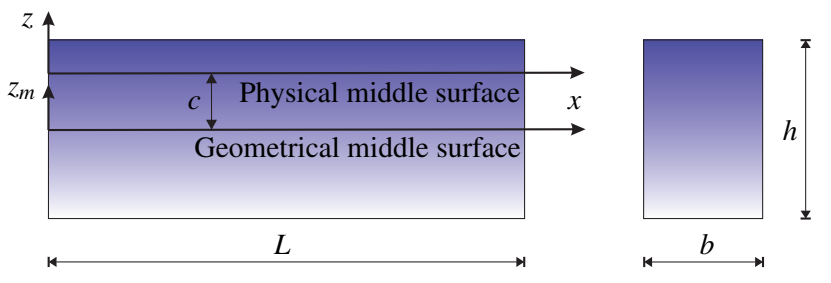

Fig. 1: Coordinate systems in physical and geometrical middle surface.

\subsection{Functionally graded material}

A small-scale FG beam of width $b$ and thickness $h$ is made of two different materials, and the effective material properties (e.g. Young's modulus $E$ and density $\rho$ ) vary continuously 


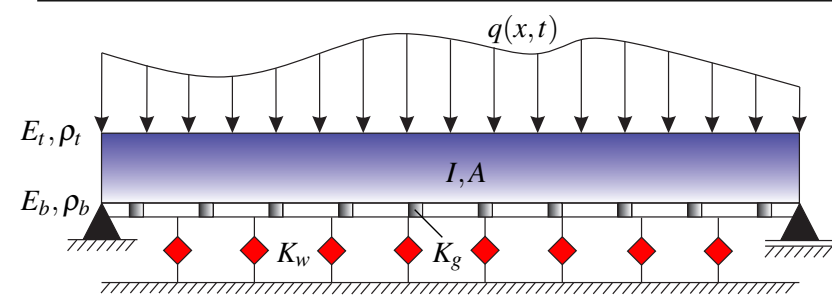

Fig. 2: Model of the nonlinear nonlocal strain gradient beam on a fractional visco-Pasternak foundation.

through the beam's thickness ( $z$ direction). Those materials properties regarding geometrical middle axis based on the power-law distribution function of material, are:

$\mathscr{P}\left(z_{m}\right)=\left(\mathscr{P}_{t}-\mathscr{P}_{b}\right)\left(\frac{z_{m}}{h}+\frac{1}{2}\right)^{k}+\mathscr{P}_{b}$,

where indices $t$ and $b$ denote the top and bottom layer of the beam and $k$ is the power-law index, which determines the material variation in the thickness direction of the beam. Geometrical and physical middle surfaces of homogeneous materials coincide. However, the change of material properties in one direction shifts the physical middle surface from the geometrical one for some finite length $c$. Such new system of reference for FG materials and structures is proposed by several authors [3],[30], [56]. Therefore, to simplify the analysis and avoid the bending-stretching mode coupling effect we will use a new coordinate system where the $x$ axis lies in the physical middle surface and the vertical axis is given as $z$, i.e.

$z=z_{m}+c$.

Constant $c$, denoting the position of the physical middle surface, can be calculated as

$c=\frac{\int_{-\frac{h}{2}}^{\frac{h}{2}} z_{m} \mathscr{P}\left(z_{m}\right) d z_{m}}{\int_{-\frac{h}{2}}^{\frac{h}{2}} \mathscr{P}\left(z_{m}\right) d z_{m}}$.

For our case of a rectangular FG beam with width $b$ and height $h$, by substituting Eq.(4) in Eq.(6), expression for $c$ is simplified to

$c=\frac{\left(E_{t}-E_{b}\right) h k}{2(2+k)\left(E_{t}+k E_{b}\right)}$.

By taking the physical middle surface as a reference, material properties can be expressed as

$E(z)=\left(E_{t}-E_{b}\right)\left(\frac{z}{h}+\frac{1}{2}\right)^{k}+E_{b}$,

$\rho(z)=\left(\rho_{t}-\rho_{b}\right)\left(\frac{z}{h}+\frac{1}{2}\right)^{k}+\rho_{b}$.

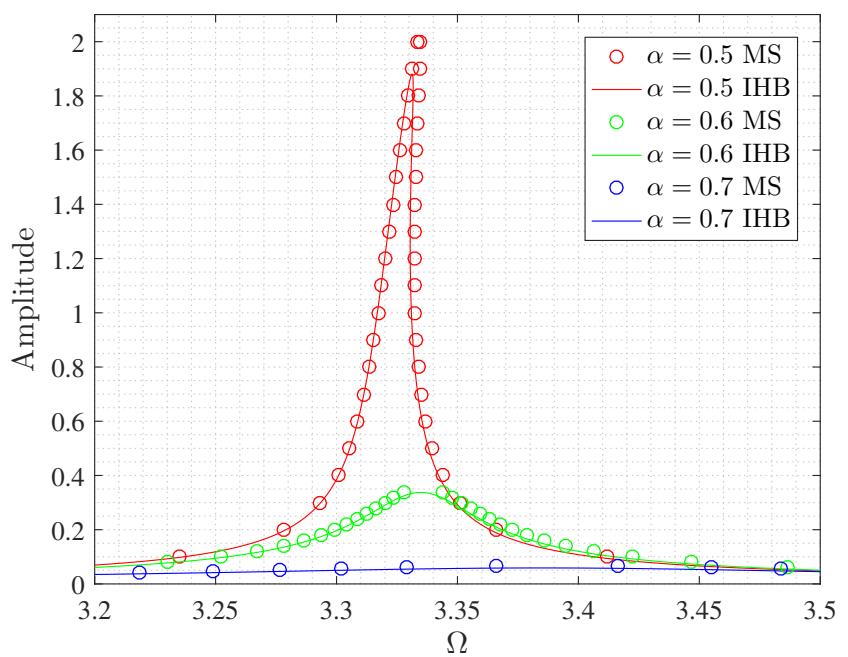

(a)

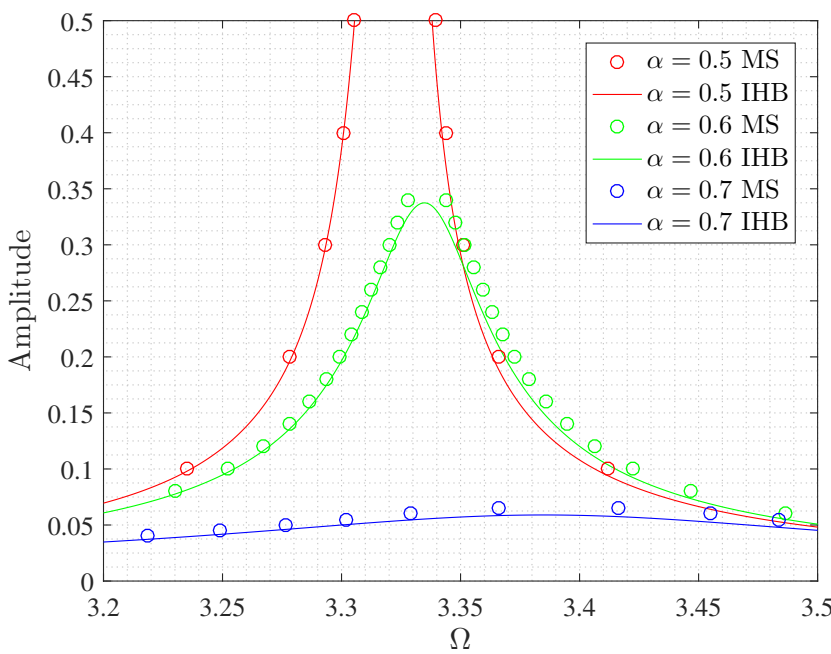

(b)

Fig. 3: The amplitude-frequency response curves of the nonlinear nonlocal strain gradient FG beam on a fractional visco-Pasternak foundation are given in sub-figure (a). The sub-figure (b) is zoomed sub-figure (a). The periodic response is obtained by the incremental harmonic balance (IHB) method - solid line and multiple scales method - circles.

\subsection{Nonlocal strain gradient theory}

According to the nonlocal strain gradient theory [36], the strain energy $U$ can be expressed as:

$U=\frac{1}{2} \int_{V}\left(\sigma_{i j} \varepsilon_{i j}+\sigma_{i j k}^{(1)} \varepsilon_{i j, k}\right) d V$,

where $\sigma_{i j}$ is the nonlocal stress, and $\sigma_{i j k}^{(1)}$ is the high-order nonlocal stress. Total stress is given by:

$t_{i j}=\sigma_{i j}-\nabla \sigma_{i j k}^{(1)}$. 


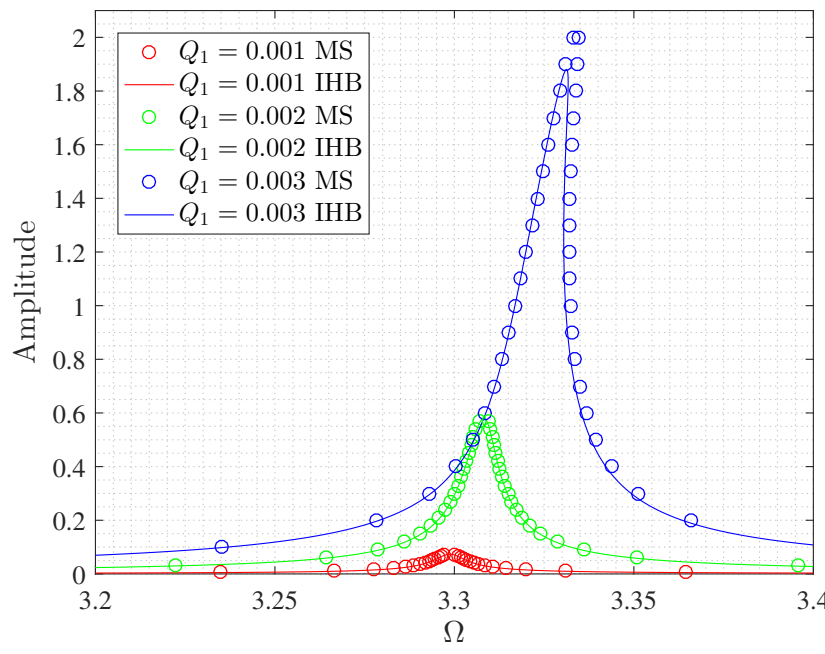

Fig. 4: The amplitude-frequency response curves of the nonlinear nonlocal strain gradient FG beam on a fractional visco-Pasternak foundation. The periodic response is obtained by the incremental harmonic balance (IHB) method - solid line and the multiple scales method - circles.

Constitutive equation for the nonlocal and local part [11] can be written as:

$$
\begin{aligned}
& \left(1-\mu^{2} \nabla^{2}\right) \sigma_{x x}=E(z) \varepsilon_{x x}, \\
& \left(1-\mu^{2} \nabla^{2}\right) \sigma_{x x x}^{(1)}=l^{2} E(z) \varepsilon_{x x, x},
\end{aligned}
$$

where $\mu$ and $l$ are the nonlocal and length scale parameter, respectively, $\nabla=\frac{\partial}{\partial x}, E(z)$ is the elasticity modulus, $\varepsilon_{x x}$ is the axial strain, and $\varepsilon_{x x, x}$ is the axial strain gradient. The general nonlocal strain gradient constitutive relation is given as [32]: $\left(1-\mu^{2} \nabla^{2}\right) t_{x x}=\left(1-l^{2} \nabla^{2}\right) E(z) \varepsilon_{x x}$.

Salehipour et al. [54] and later Batra [4] proposed the modified nonlocal theory that is applicable to non-homogenous materials. However, according to [54], when the Laplacian operator reduces to $\left(\nabla=\frac{\partial}{\partial x}\right)$ and material properties of the beam are only the functions of the thickness coordinate $z$, then classical Eringen theory can be used to account for the small-scale effects in FG beams or plates. Moreover, by introducing the physical surface reference system one can avoid coupling between the bending and stretching modes. In our analysis, we adopted both assumptions to study the nonlinear dynamic response of the FG nonlocal beam resting on the fractional visco-Pasternak foundation.

\section{Beam model and equation of motion}

Beam model is given in the Fig.(2). The displacement field of the Euler-Bernoulli beam is given as:

$u_{x}(x, z, t)=u(x, t)-z \frac{\partial w}{\partial x}, \quad u_{y}(x, z, t)=0, u_{z}(x, z, t)=w(x, t), \quad \varepsilon_{x x}=\frac{\partial u}{\partial x}+\frac{1}{2}\left(\frac{\partial w}{\partial x}\right)^{2}-z \frac{\partial^{2} w}{\partial x^{2}}$.

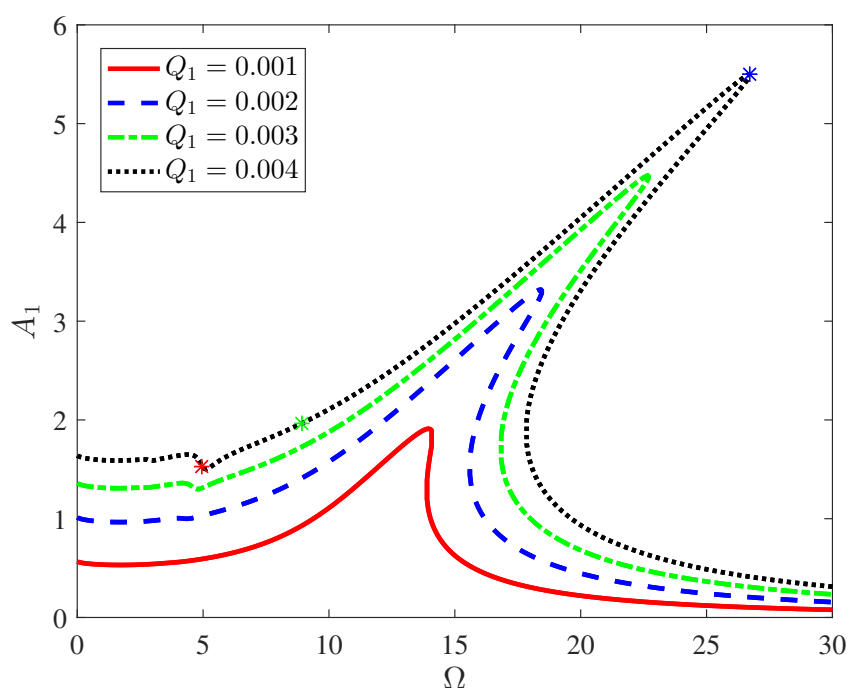

(a)

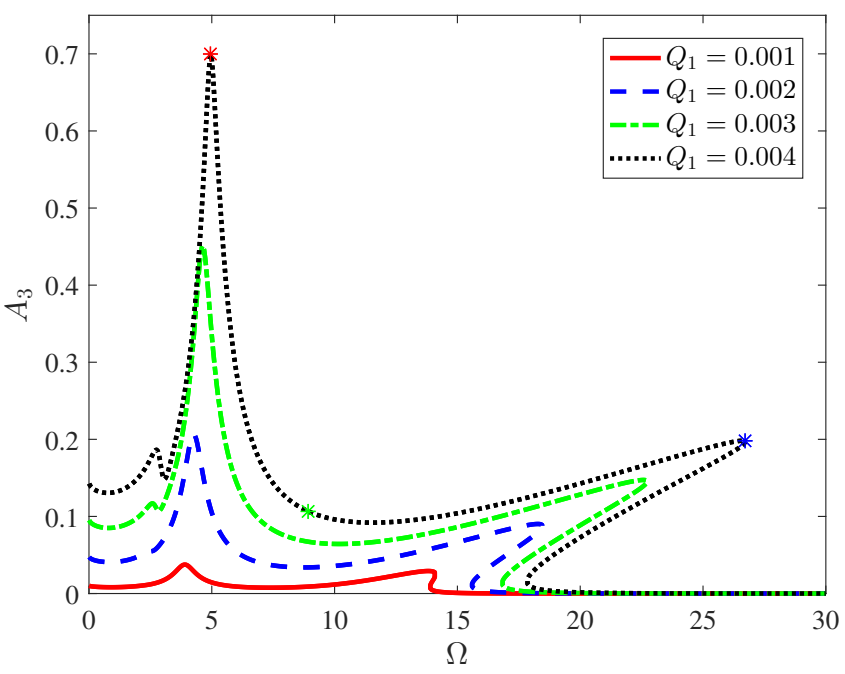

(b)

Fig. 5: The amplitude-frequency response curves $A_{1}$ - subfigure (a) and $A_{3}$ - sub-figure (b) of the nonlinear nonlocal strain gradient FG beam on fractional visco-Pasternak foundation of the amplitude $Q_{1}$.

where $u_{x}, u_{y}$ and $u_{z}$ denote the displacements along the length, width and thickness directions, respectively. Terms $u$ and $w$ are the axial and transverse displacements of the physical middle surface, respectively. Thus, the non-zero strain components of Euler-Bernoulli beam with considered geometric nonlinearity takes the form 
We are considering the following stress resultants:

$N_{x x}=\int_{A} t_{x x} d A, \quad N_{x x}^{(0)}=\int_{A} \sigma_{x x} d A, \quad N_{x x}^{(1)}=\int_{A} \sigma_{x x}^{(1)} d A$,

$M=\int_{A} z t_{x x} d A, \quad M^{(0)}=\int_{A} z \sigma_{x x} d A, \quad M^{(1)}=\int_{A} z \sigma_{x x}^{(1)} d A$.

Further, we define the extensional $A_{x x}$ and the bending coefficient $D_{x x}$ as

$\left\{A_{x x}, D_{x x}\right\}=b \int_{-\frac{h}{2}-c}^{\frac{h}{2}-c}\left\{1, z^{2}\right\} E(z) d z$.

Note that for homogeneous beam $A_{x x}=E A$ and $D_{x x}=E I$. By substituting stress resultants Eq.(17) into Eq.(14), the axial force and moment are obtained as:

$N_{x x}=N_{x x}^{(0)}-\frac{\partial N_{x x}^{(1)}}{\partial x}, \quad M=M^{(0)}-\frac{\partial M^{(1)}}{\partial x}$.

By integrating the general constitutive relation Eq.(14) over area $A$, or multiplying it with $\mathrm{z}$ and integrating over area $A$, and using relations Eq.(18) and Eq.(17) leads to

$N_{x x}=\mu^{2} \frac{\partial^{2} N_{x x}}{\partial x^{2}}+\left(1-l^{2} \frac{\partial^{2}}{\partial x^{2}}\right) A_{x x}\left(\frac{\partial u}{\partial x}+\frac{1}{2}\left(\frac{\partial w}{\partial x}\right)^{2}\right)$,

$M=\mu^{2} \frac{\partial^{2} M}{\partial x^{2}}-D_{x x}\left(1-l^{2} \frac{\partial^{2}}{\partial x^{2}}\right) \frac{\partial^{2} w}{\partial x^{2}}$.

The variation of strain energy $\delta U$ of the FG beam can be given as in [30, 57]:

$$
\begin{aligned}
\delta U= & \int_{V}\left(\sigma_{x x} \delta \varepsilon_{x x}+\sigma_{x x}^{(1)} \nabla \delta \varepsilon_{x x}\right) d V \\
= & \int_{0}^{L}\left(N_{x x} \delta \frac{\partial u}{\partial x}+N_{x x} \frac{\partial w}{\partial x} \delta \frac{\partial w}{\partial x}-M \frac{\partial^{2} w}{\partial x^{2}}\right) d x \\
& +\left[N_{x x}^{(1)} \delta \frac{\partial u}{\partial x}+N_{x x}^{(1)} \frac{\partial w}{\partial x} \delta \frac{\partial w}{\partial x}-M^{(1)} \frac{\partial^{2} w}{\partial x^{2}}\right]_{0}^{L}
\end{aligned}
$$

Virtual kinetic energy considering both the longitudinal and transverse motions can be given by

$$
\begin{aligned}
\delta K= & b \int_{0}^{L} \int_{-\frac{h}{2}-c}^{\frac{h}{2}-c} \rho(z) \frac{\partial u_{x}}{\partial t} \delta \frac{\partial u_{x}}{\partial t} d z d x \\
& +b \int_{0}^{L} \int_{-\frac{h}{2}-c}^{\frac{h}{2}-c} \rho(z) \frac{\partial u_{z}}{\partial t} \delta \frac{\partial u_{z}}{\partial t} d z d x \\
= & \int_{0}^{L} m_{0}(\dot{u} \delta \dot{u}+\dot{w} \delta \dot{w}) d x+\int_{0}^{L} m_{2} \frac{\partial \dot{w}}{\partial x} \delta \frac{\partial \dot{w}}{\partial x} d x .
\end{aligned}
$$

Note that in Eq.(23) the mass moments of inertia takes the following form

$\left\{m_{0}, m_{1}, m_{2}\right\}=b \int_{-\frac{h}{2}-c}^{\frac{h}{2}-c}\left\{1, z, z^{2}\right\} \rho(z) d z$.

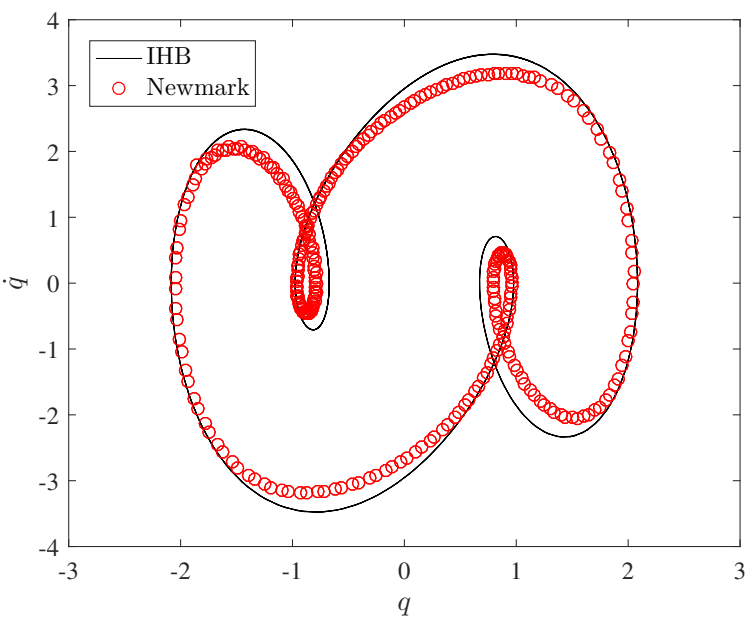

(a)

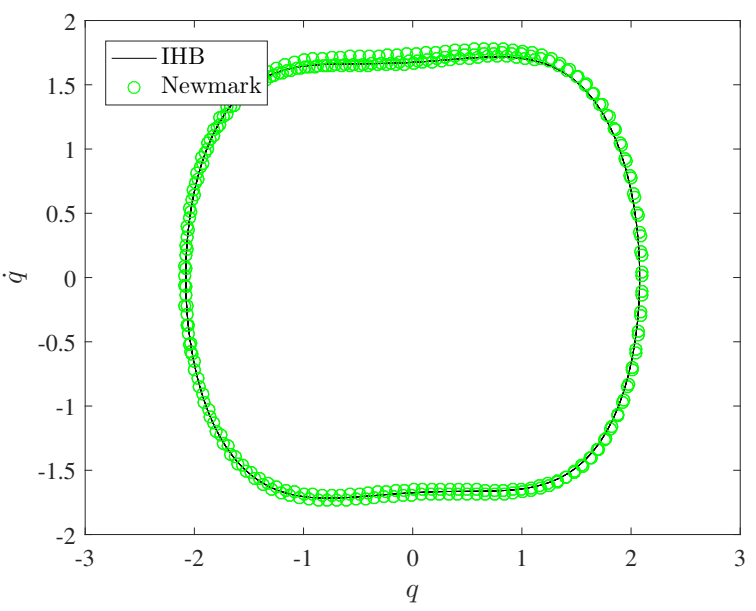

(b)

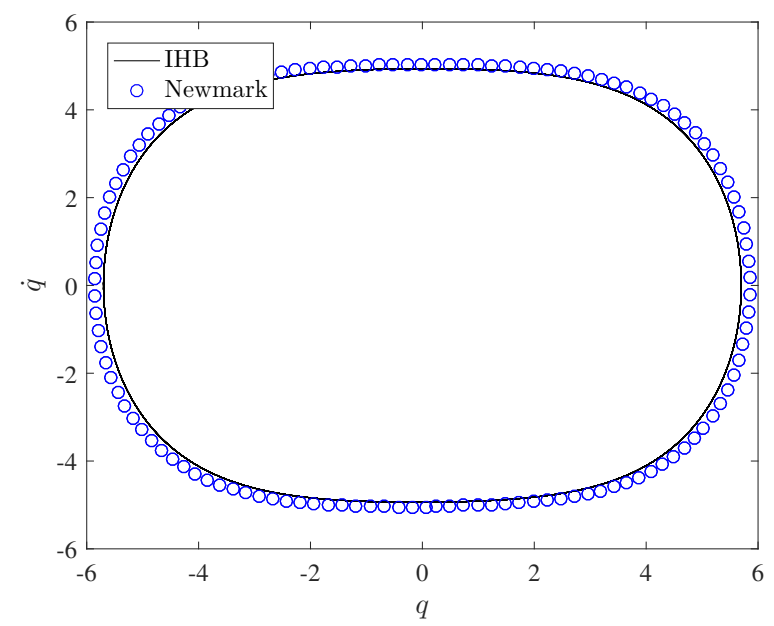

(c)

Fig. 6: The periodic response obtained by the incremental harmonic balance (IHB) method and Newmark method is taken for three different points in Fig.(5). 


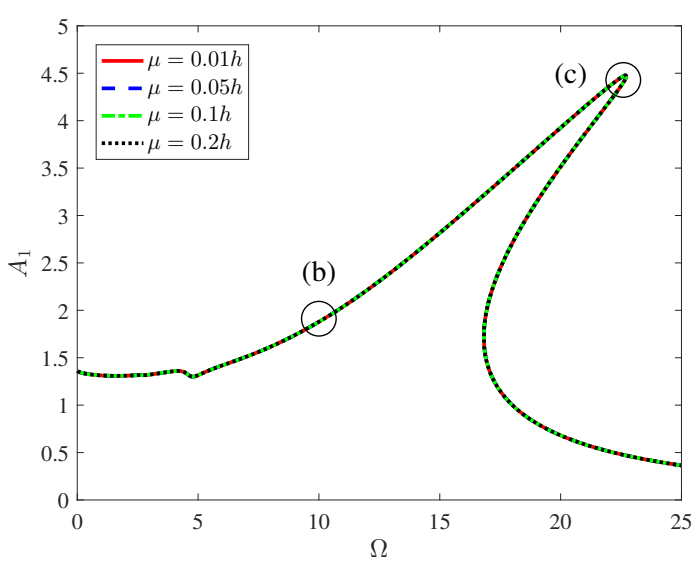

(a)

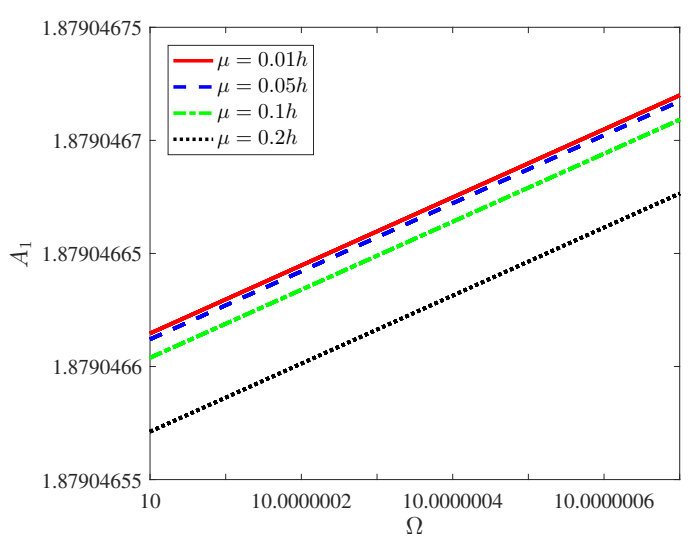

(b)

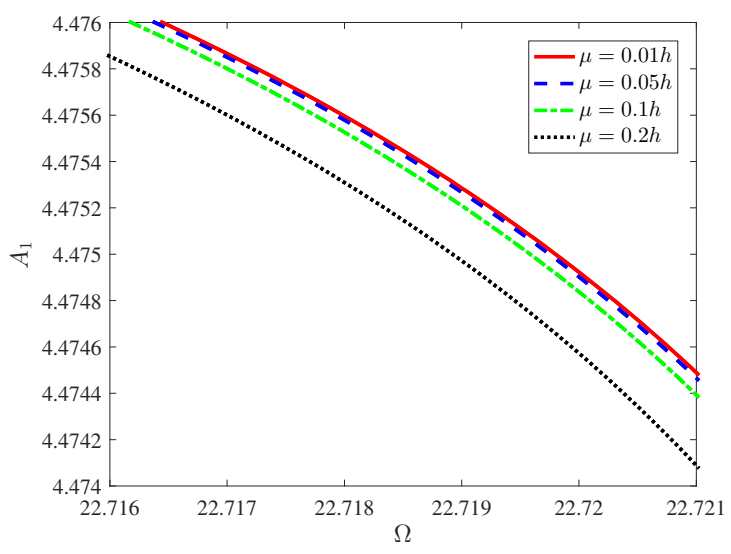

(c)

Fig. 7: The amplitude-frequency response curves of the nonlinear nonlocal strain gradient FG beam on a fractional visco-Pasternak foundation in the first harmonic amplitude $A_{1}$ and different values of the nonlocal parameter $\mu$ are given in sub-figure (a). The periodic responses obtained by the incremental harmonic balance (IHB) method are taken far from the resonant state in sub-figure (b) and close to the resonant state in sub-figure (c).
Note that for homogeneous beam $m_{0}=\rho A$ and $m_{2}=\rho I$. According to Emam and Nayfeh [9], the first-order mass moment $m_{1}$ can be neglected in the virtual kinetic energy (Eq.(23)) since its contribution is relatively small.

Virtual work of external loads can be given by [2]:

$$
\delta W=\int_{0}^{L}\left(F_{m} b+q\right) \delta w d x+\left[\bar{Q} \delta w-\bar{M} \delta \frac{\partial w}{\partial x}\right]_{0}^{L},
$$

where

$$
\begin{aligned}
F_{m} & =\left(k_{w}+K_{w} D^{\alpha}\right) w-\left(k_{g}+K_{g} D^{\alpha}\right) \frac{\partial^{2} w}{\partial x^{2}}, \\
q & =Q_{0}+Q_{1} \cos \Omega_{1} t .
\end{aligned}
$$

In Eq.(25) $F_{m}$ is the restoring force due to the visco Pasternak layer, $q$ is the distributed transverse load, $\bar{Q}$ is the external shear force, and $\bar{M}$ is the external bending moment. In Eq.(26) $D^{\alpha}$ is the operator of Riemann-Liouville fractional derivative. In [53], a similar foundation type is introduced but without the fractional time derivatives.

Hamilton's principle will be applied by using the Eq.(27):

$\int_{t_{1}}^{t_{2}}(\delta K-\delta U-\delta W) d t=0$.

By substituting Eqs.(22),(23), and (25) into Eq.(27), the following two equations of motion are obtained

$$
\begin{aligned}
& \frac{\partial N_{x x}}{\partial x}-m_{0} \frac{\partial^{2} u}{\partial t^{2}}=0 \\
& \frac{\partial^{2} M}{\partial x^{2}}+\frac{\partial}{\partial x}\left(N_{x x} \frac{\partial w}{\partial x}\right)+m_{2} \frac{\partial^{4} w}{\partial x^{2} \partial t^{2}}-m_{0} \frac{\partial^{2} w}{\partial t^{2}}-b F_{m}-q=0
\end{aligned}
$$

with classical boundary conditions at $x=0$ or $x=L$ :

$$
\begin{array}{r}
N_{x x} \text { or } u, \\
\frac{\partial M}{\partial x}+\bar{Q} \text { or } w,
\end{array}
$$

and non-classical boundary conditions at $x=0$ or $x=L$ :

$$
\begin{aligned}
& N_{x x}^{(1)} \text { or } \frac{\partial u}{\partial x} \\
& M^{(1)} \text { or } \frac{\partial^{2} w}{\partial x^{2}} .
\end{aligned}
$$

By assuming the fast dynamics, acceleration in the axial direction in Eq.(28) is negligible. Therefore $N_{x x}=C=$ const . Substituting Eqs.(14) and (16) into Eq. (17), the axial force $N_{x x}$ can be written as

$$
N_{x x}=\left(1-l^{2} \frac{\partial^{2}}{\partial x^{2}}\right) A_{x x}\left[\frac{\partial u}{\partial x}+\frac{1}{2}\left(\frac{\partial w}{\partial x}\right)^{2}\right] .
$$


By substituting Eq.(32) into Eq.(19) one can obtain

$N_{x x}^{(0)}-\frac{\partial N_{x x}^{(1)}}{\partial x}=\left(1-l^{2} \frac{\partial^{2}}{\partial x^{2}}\right) A_{x x}\left[\frac{\partial u}{\partial x}+\frac{1}{2}\left(\frac{\partial w}{\partial x}\right)^{2}\right]$

where

$N_{x x}^{(0)}=A_{x x}\left[\frac{\partial u}{\partial x}+\frac{1}{2}\left(\frac{\partial w}{\partial x}\right)^{2}\right]$

and

$N_{x x}^{(1)}=l^{2} A_{x x}\left[\frac{\partial^{2} u}{\partial x^{2}}+\frac{\partial w}{\partial x} \frac{\partial^{2} w}{\partial x^{2}}\right]$.

In the case of hinged-hinged beams, the following boundary conditions are valid:

$u(x=0)=0, \quad N^{(1)}(x=0)=0$,

$u(x=L)=0, \quad N^{(1)}(x=L)=0$.

Substituting Eq.(34) into Eq.(32) and applying the boundary conditions Eq.(36), one can obtain expression for the axial force in the following form

$N_{x x}=\frac{A_{x x}}{2 L} \int_{0}^{L}\left(\frac{\partial w}{\partial x}\right)^{2} d x$.

Substituting Eq.(37) and the second equation of motion (29) into (21) moment $M$ can be expressed as

$$
\begin{array}{r}
M=\mu^{2}\left[-\frac{A_{x x}}{2 L} \int_{0}^{L}\left(\frac{\partial w}{\partial x}\right)^{2} d x \frac{\partial^{2} w}{\partial x^{2}}-m_{2} \frac{\partial^{4} w}{\partial x^{2} \partial t^{2}}+m_{0} \frac{\partial^{2} w}{\partial t^{2}}\right. \\
\left.+b F_{m}+q\right]-D_{x x}\left(1-l^{2} \nabla^{2}\right) \frac{\partial^{2} w}{\partial x^{2}} .
\end{array}
$$

Substituting Eq.(38) in (21), we obtain the size-dependent nonlinear equations of motion for an FG Euler-Bernoulli beam model based on the nonlocal strain gradient theory

$$
\begin{aligned}
& \left(1-\mu^{2} \nabla^{2}\right)\left[-\frac{A_{x x}}{2 L} \int_{0}^{L}\left(\frac{\partial w}{\partial x}\right)^{2} d x \frac{\partial^{2} w}{\partial x^{2}}\right. \\
& \left.-m_{2} \frac{\partial^{4} w}{\partial x^{2} \partial t^{2}}+m_{0} \frac{\partial^{2} w}{\partial t^{2}}+b F_{m}+q\right] \\
& +D_{x x}\left(1-l^{2} \nabla^{2}\right) \frac{\partial^{4} w}{\partial x^{4}}=0 .
\end{aligned}
$$

After substituting relations for the external loads (Eq.(26)) in Eq.(39), it leads to Eq.(40), given by

$$
\begin{aligned}
& \left(1-\mu^{2} \nabla^{2}\right)\left[-\frac{A_{x x}}{2 L} \int_{0}^{L}\left(\frac{\partial w}{\partial x}\right)^{2} d x \frac{\partial^{2} w}{\partial x^{2}}\right. \\
& -m_{2} \frac{\partial^{4} w}{\partial x^{2} \partial t^{2}}+m_{0} \frac{\partial^{2} w}{\partial t^{2}}+b k_{w} w+b K_{w} D^{\alpha} w \\
& \left.-b k_{g} \frac{\partial^{2} w}{\partial x^{2}}-b K_{g} D^{\alpha} \frac{\partial^{2} w}{\partial x^{2}}+Q_{0}+Q_{1} \cos \Omega_{1} t\right] \\
& +D_{x x}\left(1-l^{2} \nabla^{2}\right) \frac{\partial^{4} w}{\partial x^{4}}=0 .
\end{aligned}
$$

We introduce the following nondimensional parameters:

$$
\begin{gathered}
\bar{X}=\frac{x}{L}, \quad \bar{W}=\frac{w}{k_{x}}, \quad \Sigma=\frac{l}{L}, \quad \lambda=\frac{\mu}{L}, \quad \zeta=\frac{b}{L}, \quad \tau=t S \\
\tau^{\alpha}=t^{\alpha} S^{\alpha}, \quad S=\frac{k_{x}}{L^{2}} \sqrt{\frac{A_{x x}}{m_{0}}} \quad y=\frac{m_{2}}{m_{0} L^{2}}, \quad F_{0}=\frac{Q_{0} L^{4}}{A_{x x} k_{x}^{3}} \\
F_{1}=\frac{Q_{1} L^{4}}{A_{x x} k_{x}^{3}}, \quad \Omega=\Omega_{1} \frac{L^{2}}{k_{x}} \sqrt{\frac{m_{0}}{A_{x x}}}, \quad k_{1}=\frac{\zeta k_{w} L^{5}}{A_{x x} k_{x}^{2}}, \\
K_{1}=\frac{\zeta K_{w} L^{5}}{A_{x x} k_{x}^{2}} S^{\alpha}, \quad k_{2}=\frac{\zeta k_{g} L^{3}}{A_{x x} k_{x}^{2}}, \quad K_{2}=\frac{\zeta K_{g} L^{3}}{A_{x x} k_{x}^{2}} S^{\alpha} .
\end{gathered}
$$

Note that $k_{x}$, appearing in Eq.(41), is the radius of gyration, defined in Eq.(42) as

$k_{x}=\sqrt{\frac{D_{x x}}{A_{x x}}}$.

For the homogenous beam $k_{x}=\sqrt{\frac{I_{x}}{A}}$. Using nondimensional parameters from Eqs.(41) in Eq.(40) nonlinear equation of motion is transformed into the following nondimensional form

$$
\begin{aligned}
& \left(1-\lambda^{2} \frac{\partial^{2}}{\partial \bar{X}^{2}}\right)\left[-\frac{1}{2} \int_{0}^{1}\left(\frac{\partial \bar{W}}{\partial \bar{X}}\right)^{2} d \bar{X} \frac{\partial^{2} \bar{W}}{\partial \bar{X}^{2}}\right. \\
& -y \frac{\partial^{4} \bar{W}}{\partial \bar{X}^{2} \partial \tau^{2}}+\frac{\partial^{2} \bar{W}}{\partial \tau^{2}}+k_{1} \bar{W}+K_{1} D_{\tau}^{\alpha} \bar{W} \\
& \left.-k_{2} \frac{\partial^{2} \bar{W}}{\partial \bar{X}^{2}}-K_{2} D_{\tau}^{\alpha} \frac{\partial^{2} \bar{W}}{\partial \bar{X}^{2}}+F_{0}+F_{1} \cos \Omega \tau\right] \\
& +\left(1-\Sigma^{2} \frac{\partial^{2}}{\partial \bar{X}^{2}}\right) \frac{\partial^{4} \bar{W}}{\partial \bar{X}^{4}}=0 .
\end{aligned}
$$

The solution of Eq.(43) could be assumed as a sum of products of amplitude and time functions for each mode. The most usual is single-mode discretization which has been used by many authors (For example [8, 13, 16, 18, 21, 29, $32,36,53,56,61,62])$, and a solution would be assumed as in Eq.(44). In our case, this is legitimate, since we have only cubic nonlinearity, and Nayfeh and Lacarbonara have shown in their study [45] that in certain cases one-mode Galerkin approximation fails to predict the dynamic behavior of hinged-hinged beams, especially when quadratic type nonlinearity is involved and even modes are observed in certain subharmonic or superharmonic resonance conditions.

$\bar{W}(\bar{X}, \tau)=\phi_{n}(\bar{X}) q(\tau)$,

where $\phi_{n}(\bar{X})$ is the amplitude function, $q(\tau)$ is the time function and $n=1,2, \ldots$ is the mode number. Coefficients 


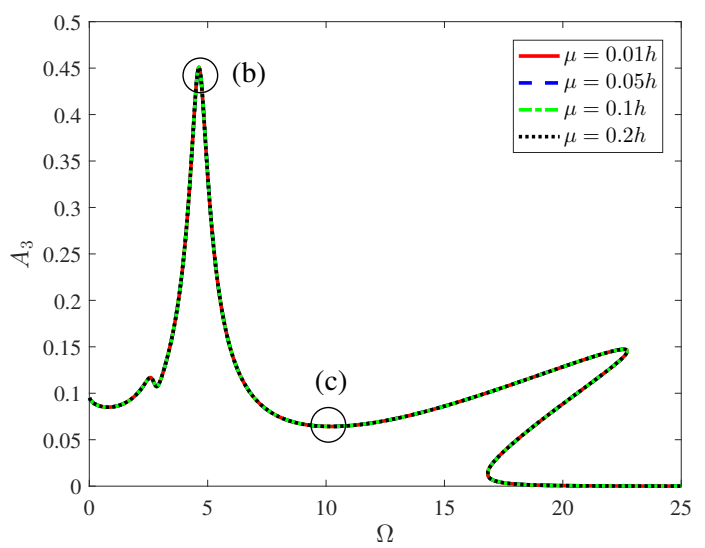

(a)

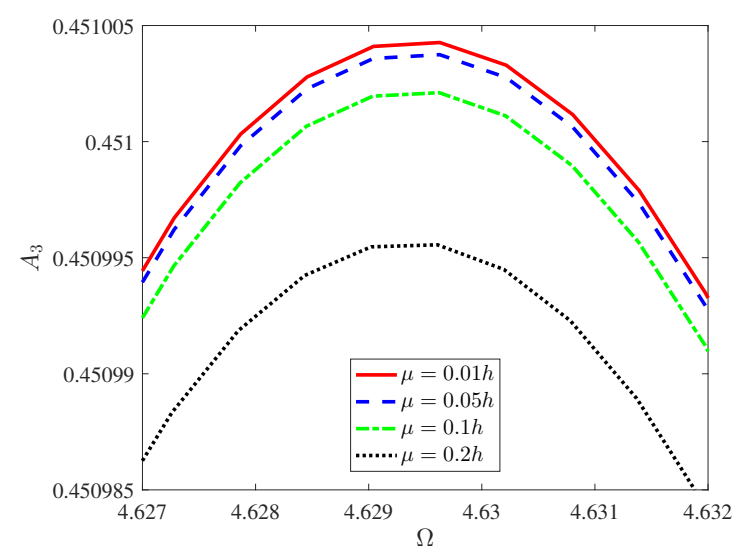

(b)

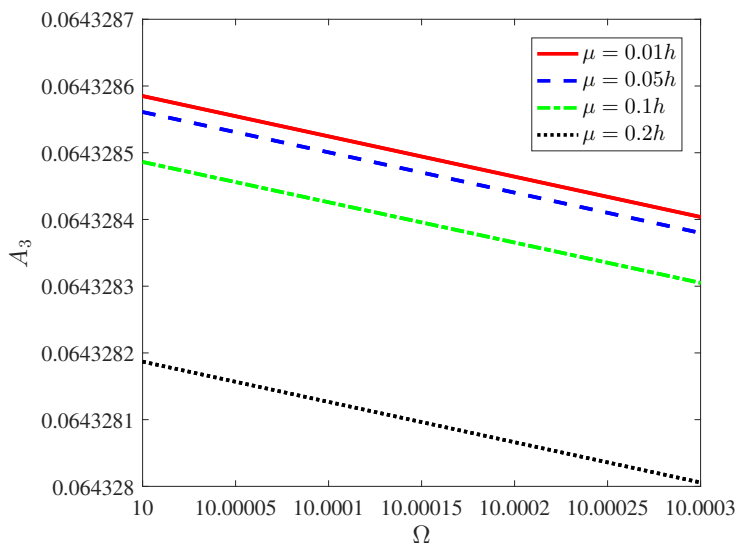

(c)

Fig. 8: The amplitude-frequency response curves of the nonlinear nonlocal strain gradient FG beam on a fractional visco-Pasternak foundation for the third harmonic amplitude $A_{3}$ for different values of the nonlocal parameter $\mu$, sub-figure (a). The periodic response obtained by the incremental harmonic balance (IHB) method is taken close to the resonant state in sub-figure (b) and far from the resonant state in sub-figure (c). $s_{0}-s_{5}$ are calculated as

$$
\left\{s_{0}, s_{1}, s_{2}, s_{3}, s_{4}, s_{5}\right\}=\int_{0}^{1}\left\{\phi, \phi^{2}, \phi^{\prime \prime} \phi, \phi^{I V} \phi, \phi^{V I} \phi, \phi^{\prime 2}\right\} d \bar{X} .
$$

By replacing Eq.(44) into Eq.(43), and using Eq.(45) we obtain the following nonlinear fractional-order differential equation

$\ddot{q}+\gamma D_{\tau}^{\alpha} q+\omega_{0}^{2} q+\theta q^{3}=f_{0}+f_{1} \cos \Omega \tau$,

where parameters are given as

$$
\begin{aligned}
\gamma & =\frac{K_{1} s_{1}-K_{2} s_{2}-\lambda^{2} K_{1} s_{2}+\lambda^{2} K_{2} s_{3}}{s_{1}-y s_{2}-\lambda^{2} s_{2}+y \lambda^{2} s_{3}}, \\
\omega_{0}^{2} & =\frac{k_{1} s_{1}-k_{2} s_{2}-\lambda^{2} k_{1} s_{2}+\lambda^{2} k_{2} s_{3}+s_{3}-\Sigma^{2} s_{4}}{s_{1}-y s_{2}-\lambda^{2} s_{2}+y \lambda^{2} s_{3}}, \\
\theta & =\frac{-\frac{1}{2} s_{5} s_{2}+\frac{1}{2} s_{5} s_{3} \lambda^{2}}{s_{1}-y s_{2}-\lambda^{2} s_{2}+y \lambda^{2} s_{3}}, \\
f_{0} & =\frac{-s_{0} F_{0}}{s_{1}-y s_{2}-\lambda^{2} s_{2}+y \lambda^{2} s_{3}}, \\
f_{1} & =\frac{-s_{0} F_{1}}{s_{1}-y s_{2}-\lambda^{2} s_{2}+y \lambda^{2} s_{3}} .
\end{aligned}
$$

Note that Eq.(40) could have been nondimensionalized in many ways. Among them, the optimal one is given in this paper. Radius of gyration $k_{x}$ (Eq.(42)) is introduced in nondimenzionalization process with the purpose to reduce nonlinear parameter $\theta$ in Eq.(46). Extreme high values of $\theta$ comparing to linear stiffness parameter $\omega_{0}^{2}$ would later induce problems with solving fractional-order differential equation of motion (Eq.(46)).

\section{Nonlinear periodic response}

Analytical perturbation methods such as the multiple scales method are usually used to solve the nonlinear fractional differential equations in the case of weak nonlinearity [55]. For strong nonlinearities, it is more common to use numerical methods such as the differential quadrature method (DQM) [40] or incremental harmonic balance (IHB) method [46]. A brief review of available numerical methods for solving the aforementioned nonlinear fractional differential equations is given by Zhou et al. [74]. In this study, periodic solutions found by the IHB method are verified with the results from both the perturbation multiple scales and Newmark numerical method.

\subsection{The incremental harmonic balance method}

IHB method has established reference quotations for solving the nonlinear structural problems. Among others, Dou and 


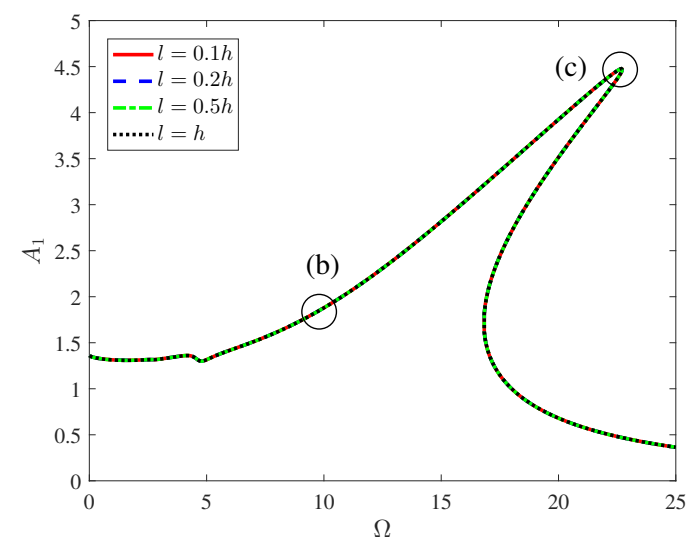

(a)

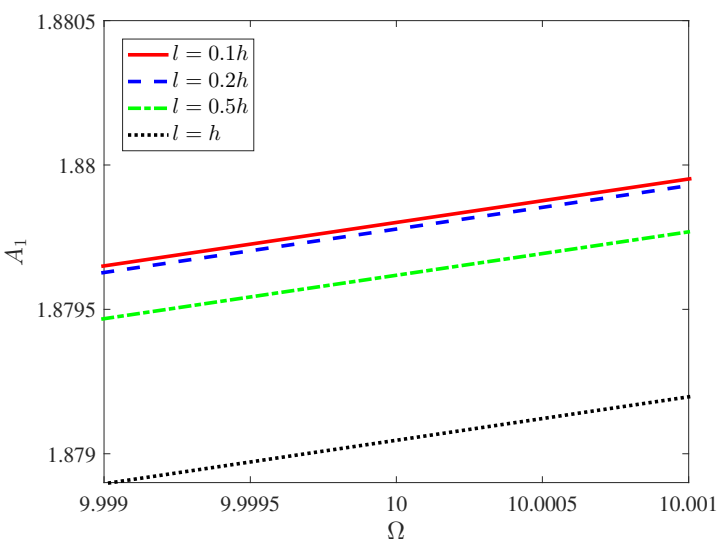

(b)

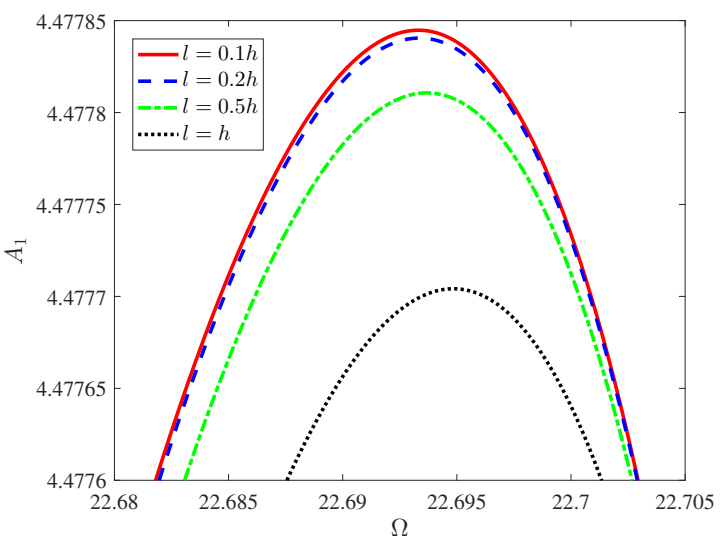

(c)

Fig. 9: The amplitude-frequency response curves of the nonlinear nonlocal strain gradient FG beam on a fractional visco-Pasternak foundation in the first harmonic amplitude $A_{1}$ and different values of the length scale parameter $l$ are given in sub-figure (a). The periodic response obtained by the IHB method and taken far from the resonant state is given in sub-figure (b) and close to the resonant state in subfigure (c).

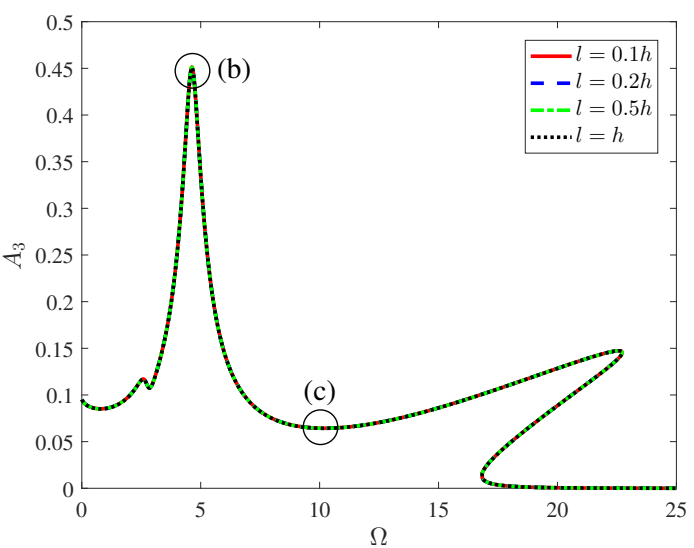

(a)

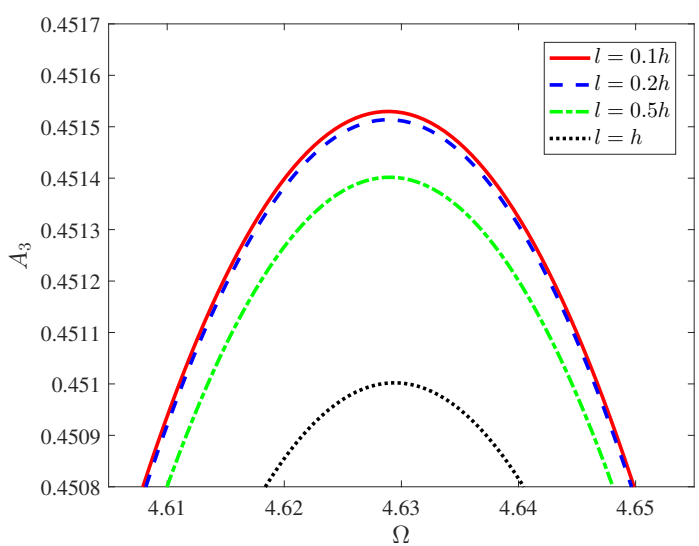

(b)

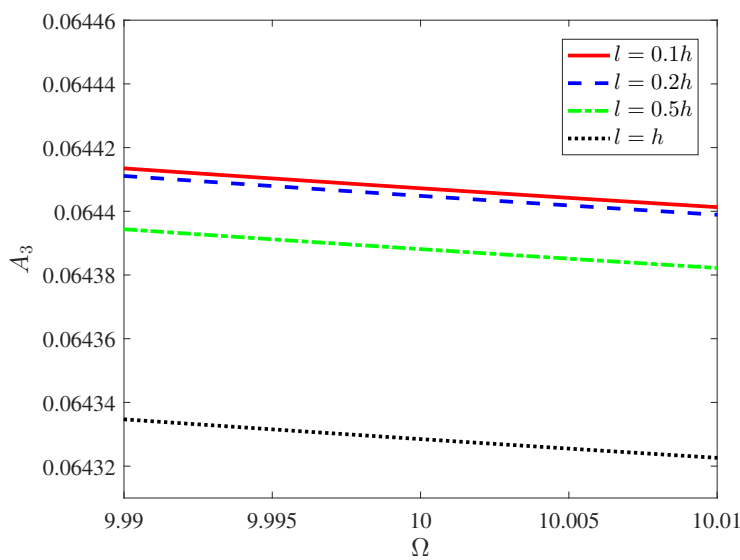

(c)

Fig. 10: The amplitude-frequency response curves of the nonlinear nonlocal strain gradient FG beam on a fractional visco-Pasternak foundation in the third harmonic amplitude $A_{3}$ for different values of the length scale parameter $l$ are given in sub-figure (a). The periodic response obtained by the IHB method taken close to resonant state is given in subfigure (b), and far from the resonant state in sub-figure (c). 
Jensen [7] developed a method for optimizing the nonlinear structural resonance with time-harmonic loads by using the IHB. Karlicic et al. [25] used IHB and perturbation method of multiple scales for investigation of the dynamic behavior and stability for a single wall carbon nanotube modeled as a nonlinear nanobeam embedded in a Kelvin-Voigt viscoelastic medium. IHB method was also employed for studying the coupled Duffing oscillators [26] and parametrically amplified Mathieu-Duffing oscillator [27] used for energy harvesting purposes.

To apply the IHB method, we introduce a new time scale $\bar{\tau}=\Omega \tau$ into Eq.(46) to obtain the system of nonlinear ordinary differential equations in the following form

$\Omega^{2} \frac{d^{2} q}{d \bar{\tau}^{2}}+\gamma \Omega^{\alpha} D_{\bar{\tau}}^{\alpha} q+\omega_{0}^{2} q+\theta q^{3}=f_{0}+f_{1} \cos \bar{\tau}$.

For the arbitrarily chosen initial values for $q_{0}$ and $\Omega_{0}$ of the steady-state modal amplitude, a neighboring state of motion has the incremental changes to the current state and can be expressed in the following form

$q=q_{0}+\Delta q, \quad \Omega=\Omega_{0}+\Delta \Omega$.

Substituting Eq.(49) into Eq.(48) and neglecting higher-order terms, we obtain a linearized incremental relation given as

$$
\begin{aligned}
& \Omega^{2} \Delta q^{\prime \prime}+\gamma \Omega_{0}^{\alpha} D_{\bar{\tau}}^{\alpha} \Delta q+\omega_{0}^{2} \Delta q+3 \theta q_{0}^{2} \Delta q \\
& \quad=r-2 \Omega_{0} q_{0}^{\prime \prime} \Delta \Omega f_{0}+f_{1} \cos \bar{\tau}
\end{aligned}
$$

where $r$ is residual term given as

$r=-\left(\Omega_{0}^{2} q_{0}^{\prime \prime}+\gamma \Omega_{0}^{\alpha} D_{\tau}^{\alpha} q_{0}+\omega_{0}^{2} q_{0}+\theta q_{0}^{3}\right)$.

To obtain the periodic solutions of the fractional-order differential equation, $q_{0}$ and $\Delta q$ are expanded as a finite Fourier series of $N$ terms as

$q_{0}=a_{0}+\sum_{n=1}^{N}\left[a_{n} \cos (n \bar{\tau})+b_{n} \sin (n \bar{\tau})\right]=C A_{0}$,

$\Delta q=\Delta a_{0}+\sum_{n=1}^{N}\left[\Delta a_{n} \cos (n \bar{\tau})+\Delta b_{n} \sin (n \bar{\tau})\right]=C \Delta A$,

where

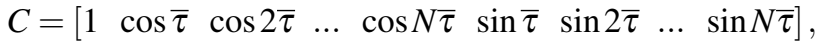

$A_{0}=\left[\begin{array}{lllllllll}a_{0} & a_{1} & a_{2} & \ldots & a_{N} & b_{1} & b_{2} & \ldots & b_{N}\end{array}\right]^{T}$,

$\Delta A=\left[\begin{array}{lllllllll}\Delta a_{0} & \Delta a_{1} & \Delta a_{2} & \ldots & \Delta a_{N} & \Delta b_{1} & \Delta b_{2} & \ldots & \Delta b_{N}\end{array}\right]^{T}$.

We substitute Eqs.(52),(53),(54),(55) and (56) into Eq.(50), and apply the Galerkin procedure. Since a fractional-order derivative is an aperiodic function, in the integration procedure we choose the time period $T=\infty$ and average the integration results for the fractional derivative. In the same way, for the periodic function, we choose the time terminal as $T=2 \pi$, which leads us to the following system of equations

$$
\begin{aligned}
& \frac{1}{2 \pi} \int_{0}^{2 \pi}(\delta \Delta q)^{T}\left[\Omega^{2} \Delta q^{\prime \prime}+\omega_{0}^{2} \Delta q+3 \theta q_{0}^{2} \Delta q\right] d \bar{\tau} \\
& +\frac{1}{T} \int_{0}^{T}(\delta \Delta q)^{T}\left[\gamma \Omega^{\alpha} D_{\bar{\tau}}^{\alpha} \Delta q\right] d \bar{\tau} \\
& =\frac{1}{2 \pi} \int_{0}^{2 \pi}(\delta \Delta q)^{T}\left[-\Omega^{2} q_{0}^{\prime \prime}-\omega_{0}^{2} q_{0}-\theta q_{0}^{3}\right. \\
& \left.\quad+f_{0}+f_{1} \cos \bar{\tau}\right] d \bar{\tau}-\frac{1}{T} \int_{0}^{T}(\delta \Delta q)^{T}\left[\gamma \Omega^{\alpha} D_{\bar{\tau}}^{\alpha} q_{0}\right] d \bar{\tau} \\
& \quad-\frac{1}{2 \pi} \int_{0}^{2 \pi}(\delta \Delta q)^{T}\left[2 \Omega_{0} q_{0}^{\prime \prime}\right] d \bar{\tau} \Delta \Omega
\end{aligned}
$$

This gives us a system of linearized algebraic equations in terms of $\Delta A$ in the following form

$M \Delta A+V \Delta \Omega=R$,

where elements of the Jacobi matrix $M$, the corrective vector $R$, and vector $V$ are given in Appendix 1 .

In case that we want the solution at a given single frequency, we would set $\Delta \Omega$ to zero in Eq.(58). Otherwise, we would solve Eq.(58) for both $A$ and $\Delta \Omega$, but insert $\Delta \Omega$ in the first entry of the vector $\Delta A$ and transform the system of equations. We initialize solution process by entering guessed values of $A$, and calculate $\Delta A$ using Eq.(58). The solution $\Delta A$ is then added to the current estimated value of $A$ to determine the new vector $A$, i.e,

$A_{k+1}=A_{k}+\Delta A$.

We repeat this process until the value of the residuum norm $|R|$ is within preset tolerance (in our case less than $10^{-5}$ ).

\subsection{The continuation method}

For starting the recurrent continuation process we need to obtain the periodic response in two successive points by using the IHB method. These initial points are usually taken far from the resonant state, where response amplitudes for both of them are having similar and small values. Then we apply the predictor-corrector method to carry out point-topoint computation for determining the corresponding branches of the frequency responses. Eq.(58) can be rewritten in the more general matrix form as

$\left[\begin{array}{ll}M & V\end{array}\right]\left[\begin{array}{c}\Delta A \\ \Delta \Omega\end{array}\right]=R$ 


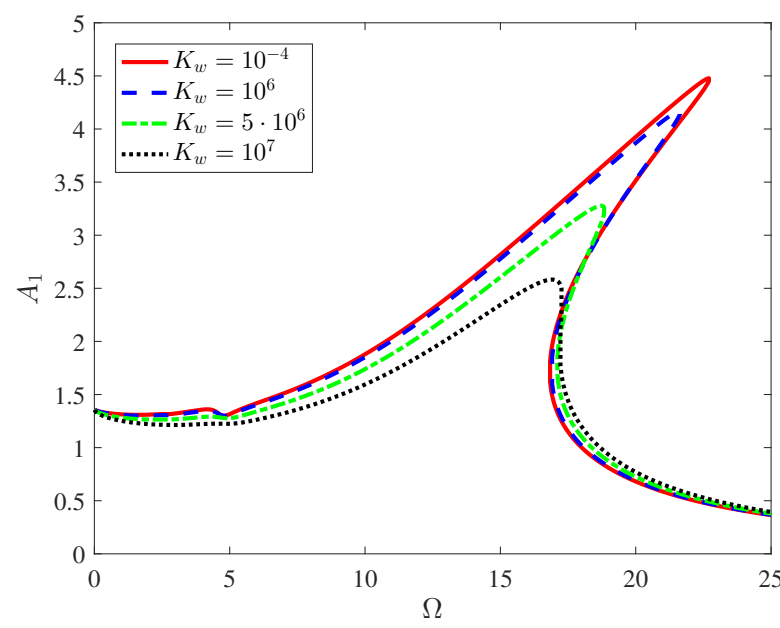

(a)

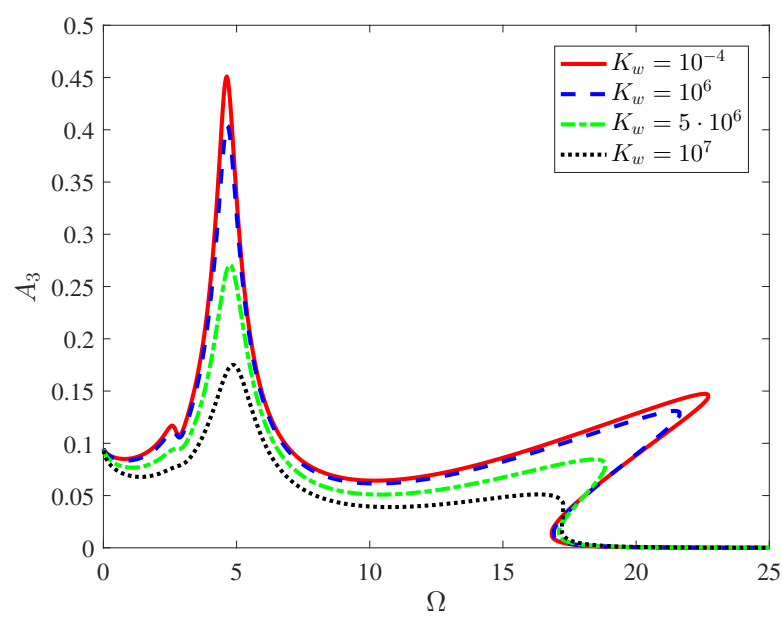

(b)

Fig. 11: The amplitude-frequency response curves of the nonlinear nonlocal strain gradient FG beam on a fractional visco-Pasternak foundation. Amplitudes $A_{1}$ (a) and $A_{3}$ (b) are plotted for different values of the parameter $K_{w}$.

We introduce new vectors $X=\left[\begin{array}{ll}A & \Omega\end{array}\right]^{T}$ and $\Delta X=\left[\begin{array}{ll}\Delta A & \Delta \Omega\end{array}\right]^{T}$. Let us also introduce a function $g(X)$ of vector $X$ in the following form: Eq.(61). Note that the function $g(X)$ can be defined in many ways, but the one given in Eq.(61) is the most appropriate

$g(X)=X^{T} X$.

We will also introduce arc-length parameter $\eta$ to follow the direction of the path. An augmented equation would be

$g(X)-\eta=0$.

The slope can be determined by using the two previous known points $X_{k-1}$ and $X_{k-2}$ on the response curves, such as

$X^{\prime}=\frac{X_{k-1}-X_{k-2}}{\left\|X_{k-1}-X_{k-2}\right\|}$.

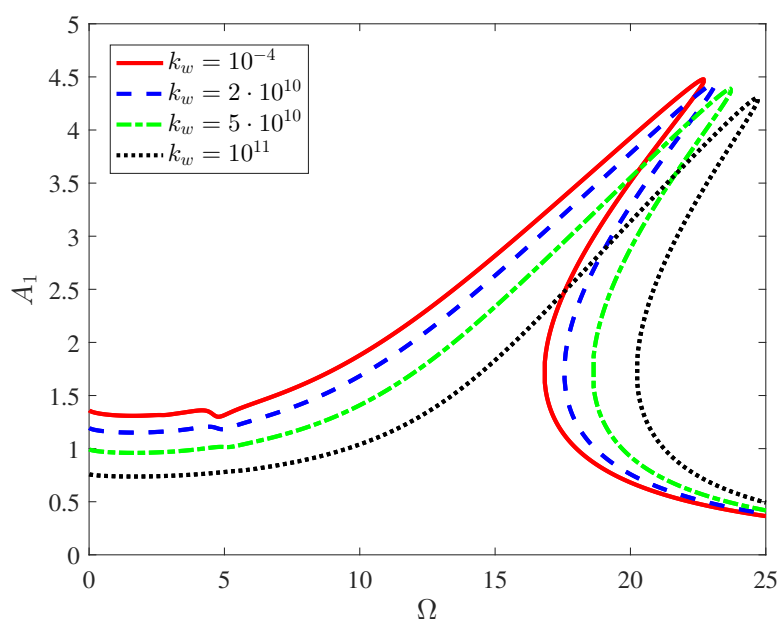

(a)

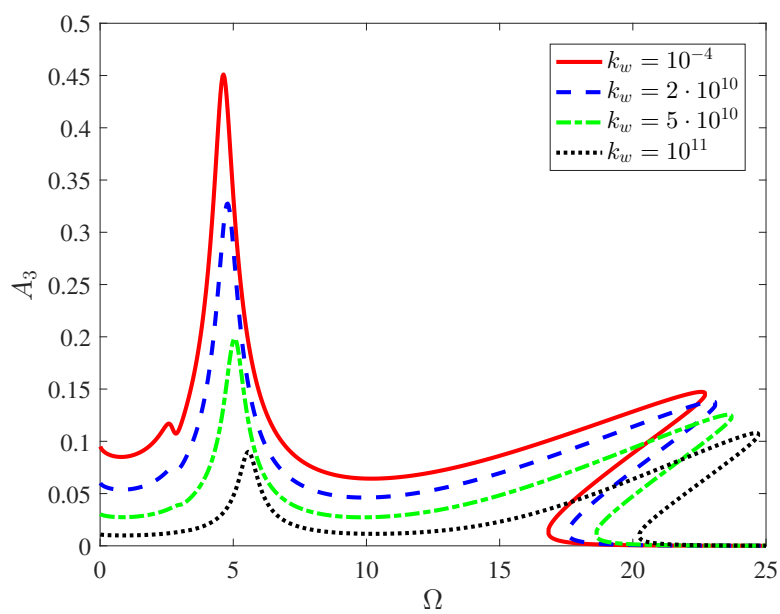

(b)

Fig. 12: The amplitude-frequency response curves of the nonlinear nonlocal strain gradient $\mathrm{FG}$ beam on a fractional visco-Pasternak foundation. Amplitudes $A_{1}$ (a) and $A_{3}$ (b) are plotted for different values of the parameter $k_{w}$.

The first prediction of the next point can be determined by

$X_{u}=X_{k-1}+\Delta \eta X^{\prime}$

Eq.(60) can be extended with Eq.(62), and then the tangent stiffness matrix and residual vector can be given in the following form

$\left[\begin{array}{cc}M & V \\ \frac{\partial g}{\partial A} & \frac{\partial g}{\partial \Omega}\end{array}\right]\left[\begin{array}{c}\Delta A \\ \Delta \Omega\end{array}\right]=\left[\begin{array}{c}R \\ \Delta \eta-g\end{array}\right]$

More information about the continuation method can be found in $[10,5,64]$. 


\section{Numerical results}

The methodology outlined in the previous section is utilized herein to find the solution of the fractional-order forced Duffing differential equation Eq.(46) and examine the resonance of a nonlocal FG beam on a fractional visco-Pasternak foundation. The combination of the IHB and path-following methods are introduced to trace branches of periodic solutions of a nonlinear model of a nonlocal strain-gradient beam on a fractional visco-Pasternak foundation with direct transversal harmonic excitation. The obtained diagrams are showing periodic responses given in the form of amplitudefrequency curves. Firstly, beam natural frequency for two different models, obtained by simplifying our model, are verified for the data available in the literature (Tables 1 and 2 ). In the second part of the numerical study, the validity of the results from the IHB method is examined (Figs. $(3,4,6)$ ), which is then followed by the parametric study in the frequency (Figs.(5,7-16)) and time-domain (Fig.(17)). It is demonstrated that the fractional visco-Pasternak layer has a significant influence on the response amplitudes. Moreover, the results obtained by the IHB method are verified with the results from multiple scales and the Newmark method. The last part of the numerical results section is devoted to the analysis of the influence of different parameters on the response. The results revealed the importance of the first and third harmonics. The parameter values of the presented mechanical model are adopted from the paper [30], extended with parameters for fractional Pasternak layer and FG material, and presented in Table 3. The static part of the excitation force $Q_{0}$ is set to zero and the dynamic part $Q_{1}$ is given in the table. When some parameter is varied remaining coefficients are taken from the Table 3. Moreover, it should be noted that the number of adopted harmonics in the Fourier series is $N=6$ and this is used in all numerical examples. The amplitudes obtained by the IHB method and corresponding to particular Fourier coefficients (Eq.(55)) and harmonics (Eq.(54)) are computed as given in Eq.(66),

$A_{0}=a_{0}, \quad A_{i}=\sqrt{a_{i}^{2}+b_{i}^{2}}, \quad(i=1,2, \ldots, N)$.

For verification with the multiple scale method, a small nondimensional bookkeeping parameter takes the value $\varepsilon=0.01$.

\subsection{Verification}

To check the derivation of the equation of motion, eigenfrequencies of two simplified models are computed and compared with results obtained by other authors. Table 1 compares the first five non-dimensional fundamental natural frequencies of a local Euler-Bernoulli beam resting on WinklerPasternak foundation for the simply supported boundary conditions with foundation parameters $k_{1}=25, k_{2}=25$ with
Table 1: The first five non-dimensional fundamental natural frequencies of a local Euler-Bernoulli beam resting on Winkler-Pasternak foundation for the simple-supported boundary conditions $\left(k_{1}=25, k_{2}=25\right)$

\begin{tabular}{ccccc}
\hline Present & Ref.[59] & Ref.[13] & Ref.[68] & Ref.[43] \\
\hline 19.2133 & 19.2133 & 19.2133 & 19.21 & 19.2178 \\
50.7002 & 50.7002 & 50.7002 & 50.7 & 50.7804 \\
100.6767 & 100.677 & 100.6767 & 100.7 & - \\
170.0281 & 170.028 & 170.0281 & 170.1 & - \\
258.9868 & 258.987 & 258.9868 & 259.1 & - \\
\hline
\end{tabular}

Table 2: Comparison of non-dimensional fundamental natural frequencies of simply supported nonlocal EulerBernoulli beam with different nonlocal parameters $\mu$ $\left(L=10, h=1, \rho=1, E=30 \cdot 10^{6}, v=0.3\right.$.)

\begin{tabular}{ccccc}
\hline$\mu$ & Present & Ref.[52] & Ref. [1] & Ref.[72] \\
\hline 0 & 9.8293 & 9.8696 & 9.8298 & 9.8696 \\
1 & 9.3774 & 9.4159 & 9.3814 & 9.4159 \\
2 & 8.9826 & 9.0195 & 8.9892 & 9.0195 \\
3 & 8.6338 & 8.6693 & 8.6424 & 8.6693 \\
4 & 8.3228 & 8.3569 & 8.3329 & 8.3569 \\
\hline
\end{tabular}

Table 3: Parameter values of the presented mechanical model

\begin{tabular}{lll}
\hline Parameter & Symbol & Value \\
\hline Fractional derivative & $\alpha$ & 0.5 \\
Young's modulus at top & $E_{t}$ & $390 \mathrm{GPa}$ \\
Young's modulus at bottom & $E_{b}$ & $210 \mathrm{GPa}$ \\
Density at top & $\rho_{t}$ & $3960 \mathrm{~kg} / \mathrm{m}^{3}$ \\
Density at bottom & $\rho_{b}$ & $7800 \mathrm{~kg} / \mathrm{m}^{3}$ \\
Power-law index & $k$ & 1 \\
Height of the beam & $h$ & $100 \mathrm{~nm}$ \\
Width of the beam & $b$ & $1 \mu \mathrm{m}$ \\
Length of the beam & $L$ & $10 \mu \mathrm{m}$ \\
Nonlocal parameter & $\mu$ & $10 \mathrm{~nm}$ \\
Length scale parameter & $l$ & $100 \mathrm{~nm}$ \\
Winkler coeff. of viscoelastic layer & $k_{w}$ & $0.0001 \mathrm{~m}$ \\
Winkler coeff. of viscoelastic layer & $K_{w}$ & $0.0001 \mathrm{Ns} / \mathrm{m}^{3}$ \\
Pasternak coeff. of viscoelastic layer & $k_{g}$ & $0.0001 \mathrm{~m}$ \\
Pasternak coeff. of viscoelastic layer & $K_{g}$ & $0.0001 \mathrm{Ns} / \mathrm{m}$ \\
Amplitude of excitation force & $Q_{1}$ & $0.003 \mathrm{~N}$ \\
\hline
\end{tabular}

values obtained by other authors [59, 13, 68, 43], where fine agreement can be observed. Besides that, we made a comparison of non-dimensional fundamental natural frequencies of simply supported nonlocal Euler beam with different values of nonlocal stress-gradient parameter $\mu$ with data available in the literature $[52,1,72]$. Results are in good agreement. 


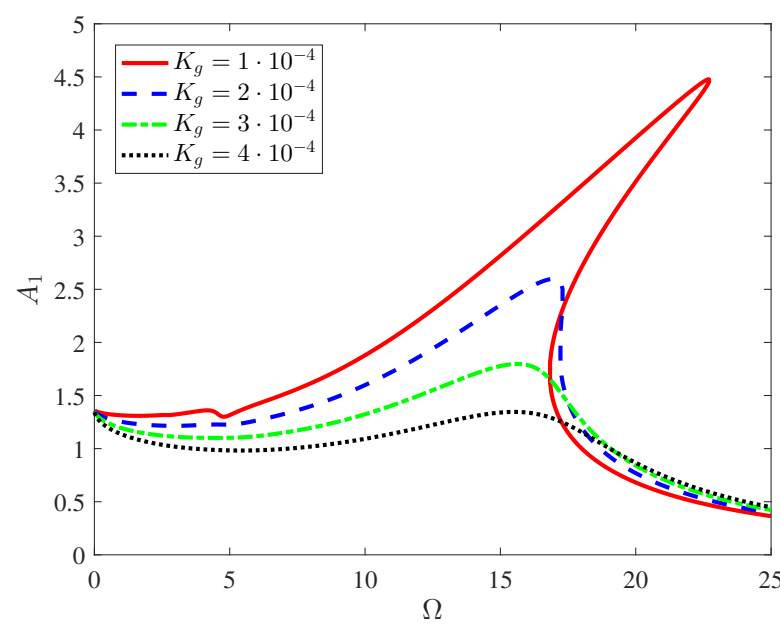

(a)

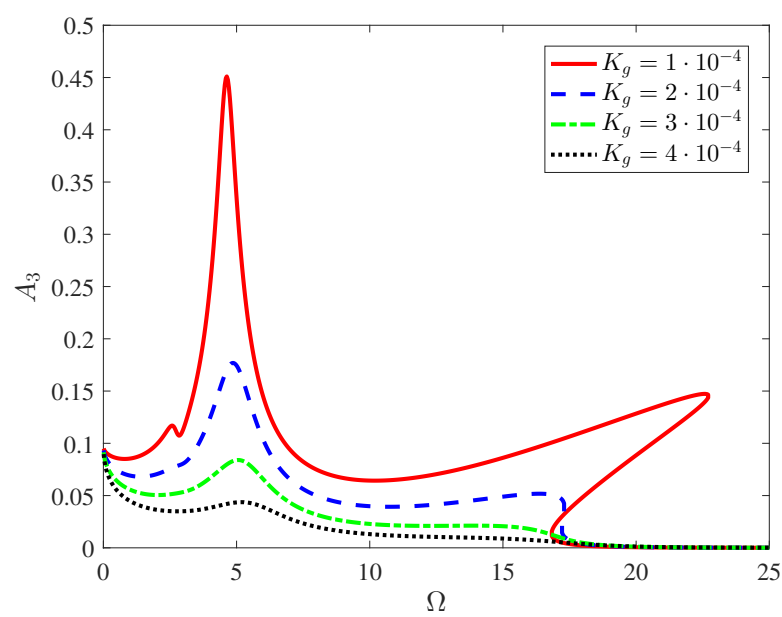

(b)

Fig. 13: The amplitude-frequency response curves of the nonlinear nonlocal strain gradient FG beam on a fractional visco-Pasternak foundation. Amplitudes $A_{1}$ (a) and $A_{3}$ (b) for different values of the parameter $K_{g}$.

With the purpose of demonstrating the reliability and accuracy of the proposed approach for the determination of the amplitude-frequency responses and corresponding periodic solutions, the obtained results from the IHB are verified with two different approaches - the perturbation method of multiple scales and the direct numerical integration by using the Newmark method. The first one is used to obtain the amplitude-frequency response diagrams, and the second one to capture periodic motions at desired excitation frequencies. The way we applied the Newmark method to solve the nonlinear fractional differential equation of motion Eq.(48) is given in Appendix 3 thoroughly.

First, we will verify the results by comparing the steadystate frequency responses for the superharmonic resonance case $\Omega=\frac{1}{3} \omega_{0}$ obtained by the IHB method with the results

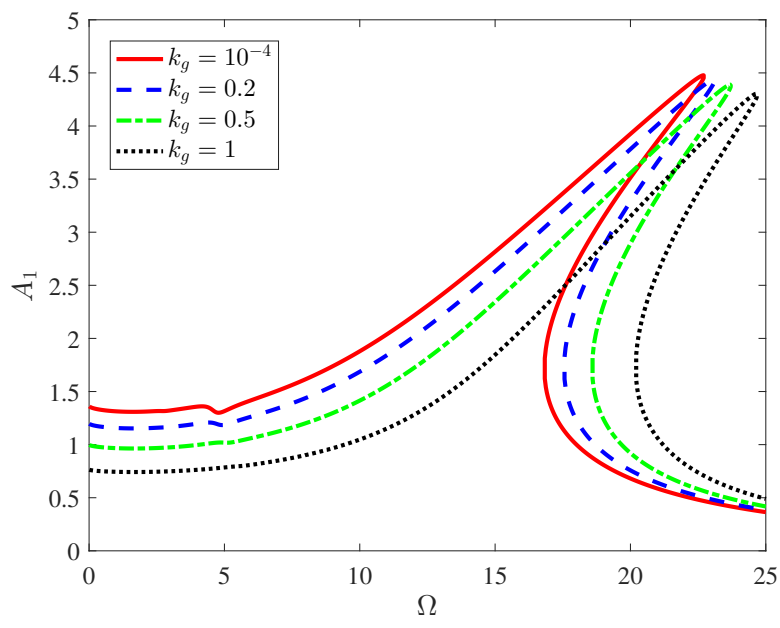

(a)

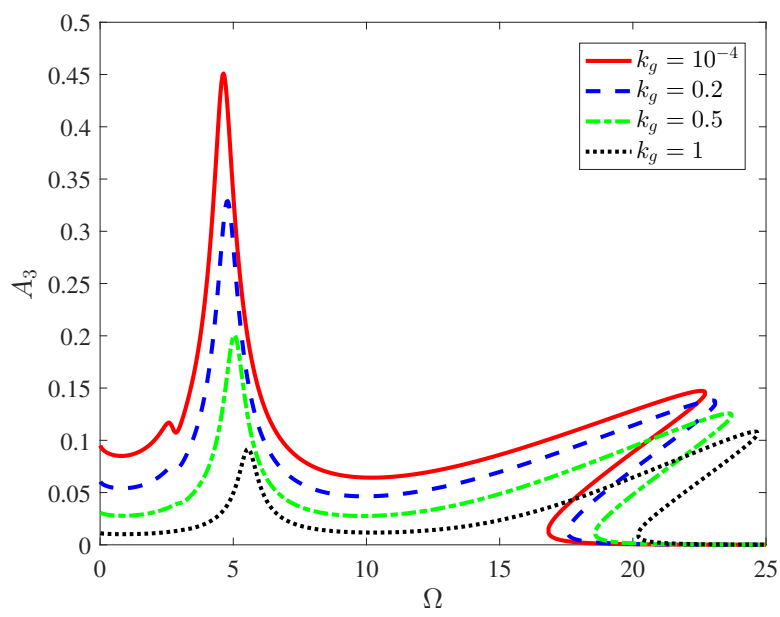

(b)

Fig. 14: The amplitude-frequency response curves of the nonlinear nonlocal strain gradient FG beam on a fractional visco-Pasternak foundation. Amplitudes $A_{1}$ (a) and $A_{3}$ (b) for different values of the parameter $k_{g}$.

from the multiple scales method, as given in Fig.(3) and Fig.(4). In these figures, response amplitudes corresponding to displacement are given on the ordinate axis while excitation frequency $\Omega$ is on the abscissa.

In Fig.(3) the amplitude-frequency response curves are given for amplitudes $A_{3}$ obtained by the IHB, and amplitudes corresponding to the excitation frequency $\Omega=\frac{1}{3} \omega_{0}$ obtained by using the multiple scales method. Fractional parametar $\alpha$ is varied. Fig.(3) (b) is zoomed Fig.(3) (a) that enables one to clearly compare the obtained results. Data in Fig.(3) reveals that results obtained by these two methods match well. Besides that, we can also observe that an increase of $\alpha$ decreases the amplitude, which is slightly shifted to the right towards higher frequencies. 
In Fig.(4) the frequency response curves are given for amplitudes $A_{3}$ obtained by using the IHB method and amplitudes corresponding to the excitation frequency $\Omega=\frac{1}{3} \omega_{0}$ obtained by using the multiple scales method. External excitation magnitudes are given as: $Q_{1}=0.001, Q_{1}=0.002$, and $Q_{1}=0.003$. From this figure, we can observe a good matching between the result obtained by two different methods. Besides that, we can also observe that an increase of the external excitation magnitude increases the amplitude and shift its value to the right towards higher frequencies.

In Fig.(5)(a) and Fig.(5)(b) the frequency response curves are given for the amplitudes $A_{1}$ and $A_{3}$, respectively, which are given on the ordinate axis while the excitation frequency $\Omega$ is on the abscissa. Due to the stiffness-hardening effect of the external excitation force parameter, not only does the maximum amplitude experience a rise but also the frequency response curves are shifted towards higher excitation frequencies. This shifting can be observed for both, the first (Fig.(5) a)) and third harmonic amplitude (Fig.(5) b)). Also, an increase of the external excitation amplitude causes a significant bending of the amplitude-frequency curves so that the multiple-value solutions may exist in the primary resonance case associated with the first and the third harmonic amplitude. Three periodic orbits are selected from the response curves (marked as star points on Fig.(5)), which are then verified with Newmark-based solutions. The periodic solutions are depicted in the phase plane, where the velocity is given on the ordinate axis while the displacement is given on the abscissa, as shown in sub-figures a), b) and c) of Fig.(6). We picked two points close and one far from the resonant state (Fig.(5)). From Fig.(6) we can observe a good matching between the result obtained by the IHB and Newmark method. However, better overlapping is achieved when we are far from the resonant state.

\subsection{Parametric study}

In the subsequent examples in this chapter, we have shown the influence of different parameters such as nonlocal parameter, strain gradient parameter, power-law index, and parameters of fractional visco-Pasternak foundation on amplitude-frequency response. The influence of excitation force is discussed in the previous subsection.

Fig.(7) and Fig.(8) show the amplitude-frequency response of the nonlinear nonlocal strain gradient FG beam on a fractional visco-Pasternak foundation with external excitation for the first $A_{1}$ and the third $A_{3}$ harmonic amplitudes, respectively, and different values of the nonlocal parameter $\mu$. In the sub-figures b) and c) are presented magnified parts of the sub-figure a). From the observation of Fig.(7) and Fig.(8), it could be found that variations in the nonlocal parameter are having weak influence in both the first and the third harmonic vibration amplitudes. Due to the large non-linearity

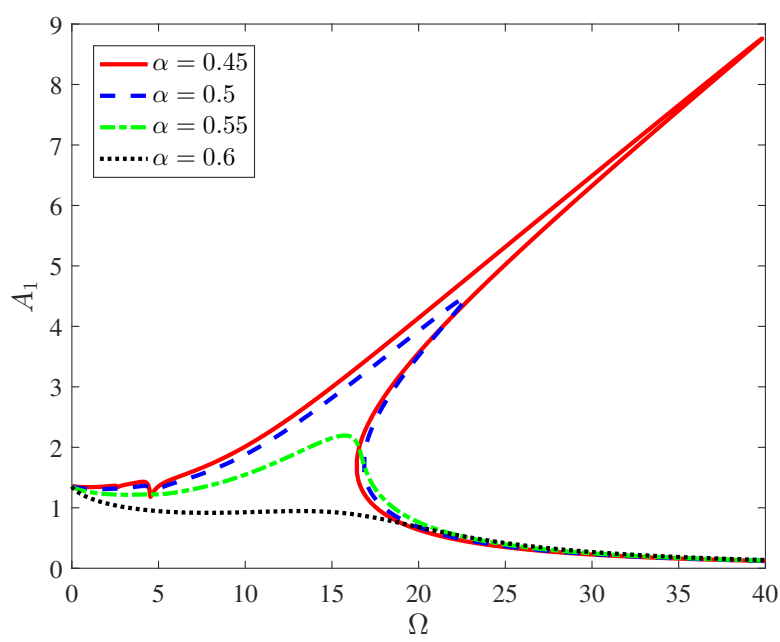

(a)

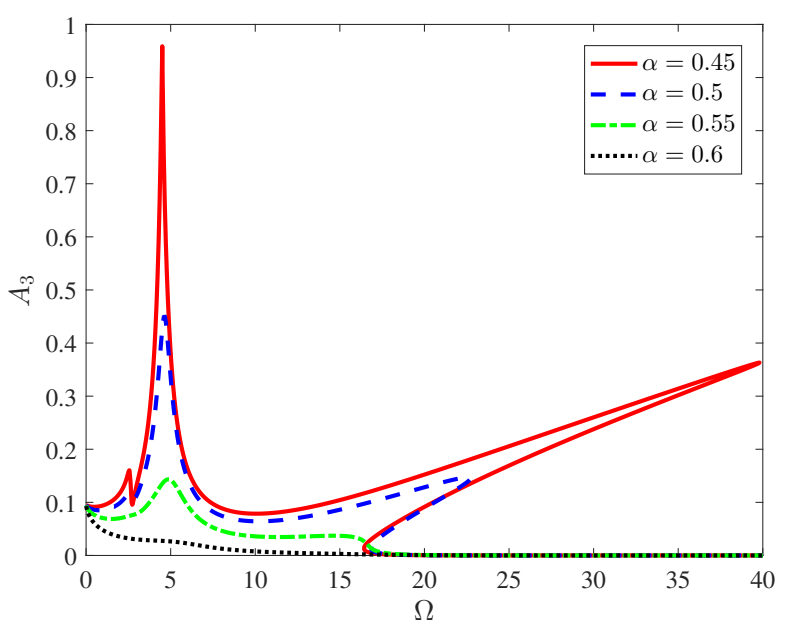

(b)

Fig. 15: The amplitude-frequency response curves of the nonlinear nonlocal strain gradient FG beam on a fractional visco-Pasternak foundation. Amplitudes $A_{1}$ (a) and $A_{3}$ (b) for different values of fractional parameter $\alpha$.

and stiffness of the system, the influence of the nonlocal parameter on amplitude-frequency response is small. In other words, nonlinearity reduces nonlocal parameter influence on the dynamic response of the system.

The amplitude-frequency response curves for different values of the length scale parameter $l$ are given in Fig.(9) and Fig.(10) for the first $A_{1}$ and the third $A_{3}$ harmonic amplitudes, respectively. In the sub-figures b) and c) are presented magnified parts of the sub-figure a). We observe that variation of the length scale parameter $l$ has a small influence on vibration amplitudes for the primary resonance case and the maximum value. Due to the large non-linearity and stiffness of the system, the influence of the length scale parameter on amplitude-frequency response is small. In other words, 


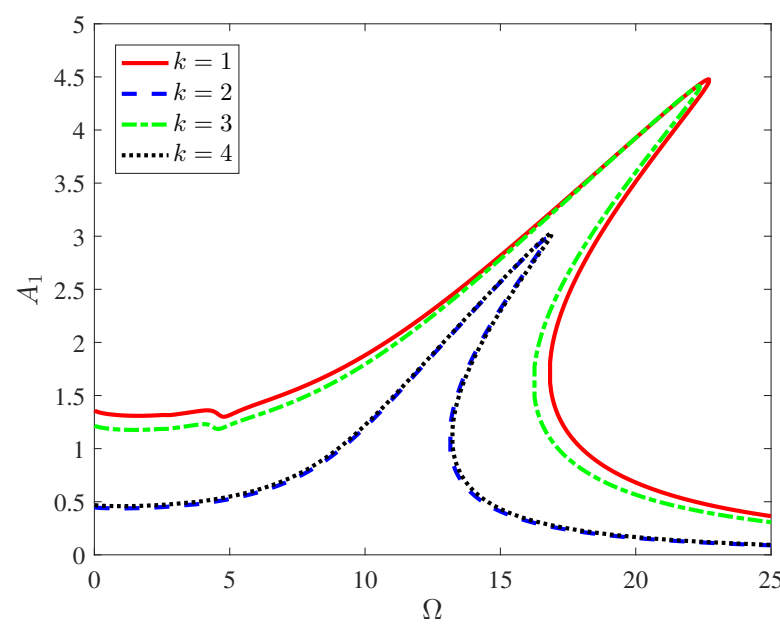

(a)

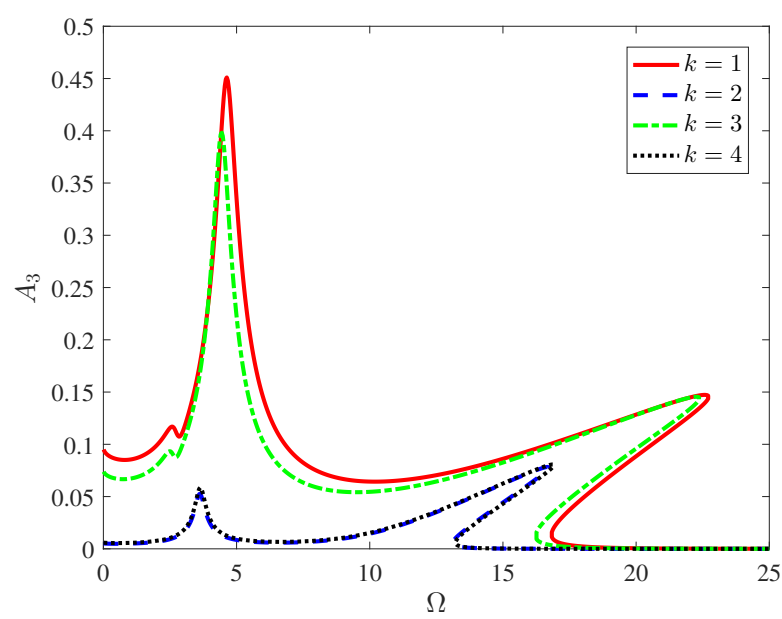

(b)

Fig. 16: The amplitude-frequency response curves of the nonlinear nonlocal strain gradient FG beam on a fractional visco-Pasternak foundation. Amplitudes $A_{1}$ (a) and $A_{3}$ (b) for different values of the power-law index $k$.

nonlinearity reduces length scale parameter influence on the dynamic response of the system.

The amplitude-frequency response curves are given for the first $A_{1}$ (Fig.(11) a)) and the third harmonic amplitude $A_{3}$ (Fig.(11) b)) and variations of the fractional visco-Pasternak foundation parameter $K_{w}$. One can observe that an increase of $K_{w}$ decreases the amplitude and therefore enlarges the total stiffness of the system. Moreover, an increase of $K_{w}$ as damping parameter decreases the natural frequencies of the system and therefore the resonance frequency is shifted to the left. Besides that, by looking at the data in-depth, it can be observed that the angle of curve tilt decreases together with the amplitude towards the curvature center for an increase of $K_{w}$, which at the same time results in weakening of the hardening type nonlinear behavior.
Fig.(12) shows the amplitude-frequency response curves for the first $A_{1}$ (Fig.(12) a)) and the third harmonic amplitude $A_{3}$ (Fig.(12) b)) and variations of the foundation parameter $k_{w}$. One can observe that an increase of $k_{w}$ decreases the amplitude with the stabilizing effect to the system vibrations and therefore the total stiffness of the system is enlarged. Besides that, an increase of $k_{w}$ as a damping parameter decreases the natural frequencies of the system and shifts the resonance frequency to the right.

The amplitude-frequency response curves in the first $A_{1}$ (Fig.(13) a)) and the third harmonic amplitude $A_{3}$ (Fig.(13) b)) are given for different values of the fractional viscoPasternak foundation parameter $K_{g}$. One can notice that an increase of the parameter $K_{g}$ decreases the resonance amplitude that is shifted to the left. This indicates that raise of $K_{g}$ augments the total stiffness of the system. Furthermore, an increase of the parameter $K_{g}$ causes weakening of the nonlinear hardening behavior of the response. Namely, the hardening-type nonlinearity becomes more apparent when the damping parameter $K_{g}$ is small.

Fig.(14) shows the amplitude-frequency response curves for the first $A_{1}$ (Fig.(14) a)) and the third harmonic amplitude $A_{3}$ (Fig.(14) b)) for different values of the foundation parameter $k_{g}$. One can notice that an increase of the parameter $k_{g}$ decreases the resonance amplitude that is shifted to the right significantly enlarging the hardening effects of nonlinearity. This indicates that raise of $k_{g}$ increases the total stiffness of the system.

By comparing the variation of $K_{w}, k_{w}, K_{g}, k_{g}$, one can observe that an increase of the parameter $K_{g}$ has a bigger influence on the increasing total stiffness of the system than the parameter $K_{w}$, even though both parameters contribute to the amplitude decrease. However, $k_{g}$ and $k_{w}$ have similar effects of moving the amplitude-frequency curve to the right towards the higher values of the external frequency with the light reduction of amplitude values.

The amplitude-frequency response curves in the first $A_{1}$ (Fig.(15) a)) and the third harmonic amplitude $A_{3}$ (Fig.(15) b)) are given for different values of the fractional-order derivative $\alpha$ in the model of visco-Pasternak foundation. It can be noticed that a decrease of the fractional-order parameter $\alpha$ by a step of 0,05 increases the amplitude values by almost double in the primary resonance case. This significant influence of the parameter $\alpha$ is caused by damping features of the system become less pronounced due to the elastic-like behavior of the fractional term. Moreover, a decrease of the fractional derivative parameter $\alpha$ makes the equivalent stiffness coefficient larger, which results in the rightwards bending of the amplitude-frequency curves and larger primary resonance frequencies.

Amplitudes of the first $A_{1}$ (Fig.(16) a)) and the third harmonic $A_{3}$ (Fig.(16) b)) of the amplitude-frequency response are given for different values of the power-law index $k$ that 
defines the FG material. One can notice that for $k=1$ and $k=3$ the resonant frequencies and hysteresis domain becomes larger and more shifted and bent towards the positive direction of lateral axis than for the case when $k=2$ and $k=4$. This can be attributed to increased stiffness properties of the nonlocal beam for these uneven values of the power-law index that increases the hardening nonlinearity and stiffness features of the system.

\subsection{Time response}

In this section, we show the time responses of the system obtained via the Newmark method. The influences of the fractional-order derivative parameter $\alpha$ (Fig.(17)) are studied to show their effect on the time-dependent behavior of the system. To understand the influence of the fractional visco-Pasternak layer on the initial harmonic excitation of the beam, the following initial conditions are adopted $q(0)=$ $1, \dot{q}(0)=1$. We adopted the following values of fractional parameter $\alpha=0.5,0.6,0.7$. The dimensionless time period $\mathrm{T}=200$ is used in the simulation. Similar conclusions can be drawn here as for the amplitude-frequency response. One can observe that an increase of the fractional parameter $\alpha$ leads to stronger damping in time and reduced and attenuated amplitudes of the response. Also, a weak beating phenomenon with decreasing intervals in time can be observed.

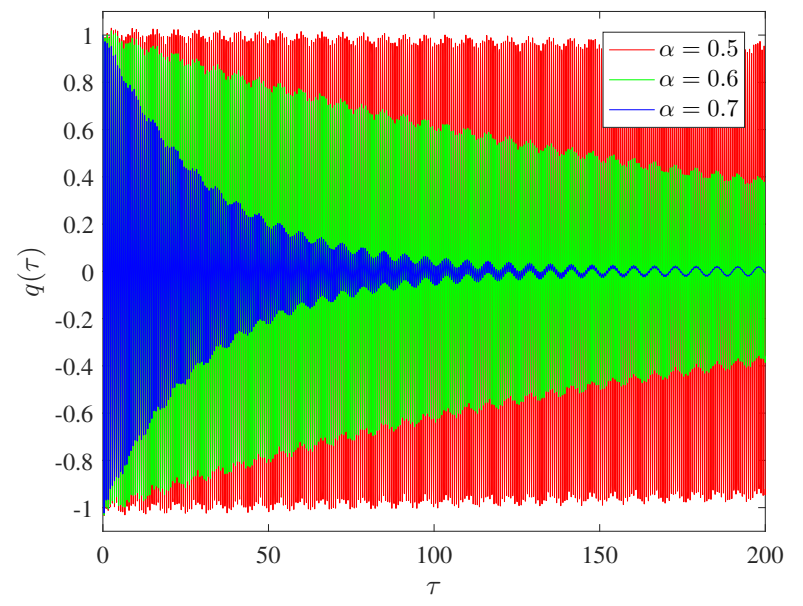

(a)

Fig. 17: The time response curves of the nonlinear nonlocal strain gradient FG beam on a fractional visco-Pasternak foundation. Amplitudes for different values of the fractional derivative parameter.

\subsection{Summary of the numerical results}

The following conclusions can be drawn about the results presented in this section. The hardening-type nonlinearity becomes more apparent when the force increases and the following parameters decrease: nonlocal parameter $\mu$, straingradient length scale parameter $l$, parameters of visco-Pasternak foundation $\alpha, K_{w}, K_{g}$. If we increase the external excitation amplitude in this system, the primary resonances will be strengthened and shifted rightwards i.e. towards higher excitation frequencies. In this case, the hysteresis domain would also increase. Nonlocal and length scale parameters are both having a small influence on the amplitude-frequency response. Parameters of the visco-Pasternak foundation $K_{w}$ and $K_{g}$ augments the total stiffness of the system since their increasing cause response amplitudes decreasing. Specifically, foundation parameters $K_{w}$ and $k_{w}$ have smaller influence on amplitude-frequency response comparing to parameters $K_{g}$ and $k_{g}$. The even values of power-law index $k$ causes higher amplitude values in comparison to their odd values.

In addition, we remark on the mutual interactions of the regime in the time domain of the single-amplitude mode of beam vibration. This is observed from the amplitudefrequency diagrams of the first mode $A_{1}$, Figs. 7, 9-16, a small jump in amplitude in the region of external frequency about 5, before the resonant region, which corresponds to the contribution of resonant jumps of the third amplitude $A_{3}$. The amplitude of the third mode $A_{3}$ has a resonant range around this frequency and their values go up to values that can be registered for these small jumps on the amplitude diagrams of the first mode $A_{1}$. The second resonant region of the third amplitude $A_{3}$ is in the same frequency domain as the one of the first amplitude $A_{1}$, the interval $15-25$. Thus, the changes of the $A_{1}$ diagram from this interval are contributed also by the behavior of the third amplitude $A_{3}$ in this interval.

\section{Conclusions}

In this paper, we analyzed the nonlinear vibration problem of a nonlocal beam resting on the fractional visco-Pasternak foundation by using the nonlocal strain-gradient theory and fractional order damping. The governing equation is derived by using Hamilton's principle and then discretized via the Galerkin approximation, which yields a corresponding nonlinear fractional-order forced Duffing type differential equation. The solution is sought for the steady-state superharmonic resonance conditions by using the perturbation multiple time scales method for the weakly nonlinear case and IHB and Newmark method for the strongly nonlinear case. From the verification study, it is revealed that the IHB method is in good agreement with the multiple time scales analysis 
for the weakly nonlinear case and with the numerical Newmark method for the strongly nonlinear case. The main advantage of the IHB method over the multiple scales method lies in the fact that it does not require an introduction of small parameter and thus strong nonlinearity cases can be observed. On the other side, the superiority of the IHB over the Newmark approach is the simple computational implementation and easier determination of periodic solutions. We have also shown that the introduction of the IHB method in the analysis of NLSGT structures can lead to more reliable studies of strongly nonlinear systems. In our parametric study, we concluded that the nonlocal and length scale parameters are having a small influence on the amplitudefrequency response. On the other hand, parameters of the visco-Pasternak foundation remarkably affect the response amplitudes. Finally, the power-law index displays a significant effect on the frequency response, which was also discussed in the numerical analysis. Generally speaking, the system vibration amplitudes are higher for the odd values of the power-law index comparing to materials with the even values of this parameter.

Acknowledgements This research was sponsored by the Serbian Ministry of Education, Science, and Technological Development. D.K. and M.C were funded by the Marie Skłodowska-Curie Actions - European Commission fellowship (grant number 799201- METACTIVE, and grant number 896942-METASINK, respectively).

\section{Conflict of interest}

The authors declare that they have no conflict of interest.

\section{References}

[1] Reza Ansari et al. "A sixth-order compact finite difference method for vibrational analysis of nanobeams embedded in an elastic medium based on nonlocal beam theory". In: Mathematical and Computer Modelling 54.11-12 (2011), pp. 2577-2586.

[2] AR Askarian, MR Permoon, and M Shakouri. "Vibration analysis of pipes conveying fluid resting on a fractional Kelvin-Voigt viscoelastic foundation with general boundary conditions". In: International Journal of Mechanical Sciences (2020), p. 105702.

[3] Raffaele Barretta et al. "Functionally graded Timoshenko nanobeams: a novel nonlocal gradient formulation". In: Composites Part B: Engineering 100 (2016), pp. 208-219.

[4] RC Batra. "Misuse of Eringen's nonlocal elasticity theory for functionally graded materials". In: International Journal of Engineering Science 159 (), p. 103425.
[5] Udbhau Bhattiprolu, Anil K Bajaj, and Patricia Davies. "Periodic response predictions of beams on nonlinear and viscoelastic unilateral foundations using incremental harmonic balance method". In: International Journal of Solids and Structures 99 (2016), pp. 2839.

[6] Oreste S Bursi and P-SB SHING. "Evaluation of some implicit time-stepping algorithms for pseudodynamic tests". In: Earthquake engineering \& structural dynamics 25.4 (1996), pp. 333-355.

[7] Suguang Dou and Jakob Søndergaard Jensen. "Optimization of nonlinear structural resonance using the incremental harmonic balance method". In: Journal of Sound and Vibration 334 (2015), pp. 239-254.

[8] Sami El-Borgi, Ralston Fernandes, and JN Reddy. "Non-local free and forced vibrations of graded nanobeams resting on a non-linear elastic foundation". In: International Journal of Non-Linear Mechanics 77 (2015), pp. 348-363.

[9] Samir A Emam and Ali H Nayfeh. "Postbuckling and free vibrations of composite beams". In: Composite Structures 88.4 (2009), pp. 636-642.

[10] Sebastian Engelnkemper et al. "Continuation for thin film hydrodynamics and related scalar problems". In: Computational Modelling of Bifurcations and Instabilities in Fluid Dynamics. Springer, 2019, pp. 459501.

[11] A Cemal Eringen. "On differential equations of nonlocal elasticity and solutions of screw dislocation and surface waves". In: Journal of applied physics 54.9 (1983), pp. 4703-4710.

[12] Georgios I Evangelatos and Pol D Spanos. "An accelerated Newmark scheme for integrating the equation of motion of nonlinear systems comprising restoring elements governed by fractional derivatives". In: Recent advances in mechanics. Springer, 2011, pp. 159177.

[13] Guy Joseph Eyebe et al. "Nonlinear vibration of a nonlocal nanobeam resting on fractional-order viscoelastic Pasternak foundations". In: Fractal and Fractional 2.3 (2018), p. 21.

[14] Yin Fan, Yang Xiang, and Hui-Shen Shen. "Nonlinear dynamics of temperature-dependent FG-GRC laminated beams resting on visco-Pasternak foundations". In: International Journal of Structural Stability and Dynamics 20.01 (2020), p. 2050012.

[15] Yang Gao, Wanshen Xiao, and Haiping Zhu. "Nonlinear vibration of functionally graded nano-tubes using nonlocal strain gradient theory and a two-steps perturbation method". In: Struct. Eng. Mech 69.2 (2019), pp. 205-219.

[16] Majid Ghadiri and S Hamed S Hosseini. "Parametric excitation of pre-stressed graphene sheets under mag- 
netic field: nonlinear vibration and dynamic instability”. In: International Journal of Structural Stability and Dynamics 19.11 (2019), p. 1950135.

[17] Mergen H Ghayesh and Ali Farajpour. "A review on the mechanics of functionally graded nanoscale and microscale structures". In: International Journal of Engineering Science 137 (2019), pp. 8-36.

[18] Mohammad Hashemian et al. "Nonlocal dynamic stability analysis of a Timoshenko nanobeam subjected to a sequence of moving nanoparticles considering surface effects". In: Mechanics of Materials (2020), p. 103452.

[19] XQ He, S Kitipornchai, and KM Liew. "Resonance analysis of multi-layered graphene sheets used as nanoscale resonators". In: Nanotechnology 16.10 (2005), p. 2086.

[20] Elmer S Hung and Stephen D Senturia. "Extending the travel range of analog-tuned electrostatic actuators". In: Journal of microelectromechanical systems 8.4 (1999), pp. 497-505.

[21] Ilgar Jafarsadeghi-Pournaki et al. "Size-dependent dynamics of a FG nanobeam near nonlinear resonances induced by heat". In: Applied Mathematical Modelling (2020).

[22] MH Jalaei, A Ghorbanpour Arani, and H NguyenXuan. "Investigation of thermal and magnetic field effects on the dynamic instability of FG Timoshenko nanobeam employing nonlocal strain gradient theory". In: International Journal of Mechanical Sciences 161 (2019), p. 105043.

[23] G Janevski, N Despenić, and I Pavlović. "Thermal buckling and free vibration of Euler-Bernoulli FG nanobeams based on the higher-order nonlocal strain gradient theory." In: Archives of Mechanics 72.2 (2020).

[24] Goran Janevski, Ivan Pavlović, and Nikola Despenić. "Thermal buckling and free vibration of Timoshenko FG nanobeams based on the higher-order nonlocal strain gradient theory". In: Journal of Mechanics of Materials and Structures 15.1 (2020), pp. 107-133.

[25] Danilo Karličić et al. "Dynamic stability of singlewalled carbon nanotube embedded in a viscoelastic medium under the influence of the axially harmonic load". In: Composite Structures 162 (2017), pp. 227243.

[26] Danilo Karličić et al. "Nonlinear energy harvester with coupled Duffing oscillators". In: Communications in Nonlinear Science and Numerical Simulation (2020), p. 105394.

[27] Danilo Karličić et al. "Parametrically amplified MathieuDuffing nonlinear energy harvesters". In: Journal of Sound and Vibration (2020), p. 115677.

[28] David CC Lam et al. "Experiments and theory in strain gradient elasticity". In: Journal of the Mechanics and Physics of Solids 51.8 (2003), pp. 1477-1508.
[29] Roman Lewandowski and Przemysław Wielentejczyk. "Nonlinear vibration of viscoelastic beams described using fractional order derivatives". In: Journal of Sound and Vibration 399 (2017), pp. 228-243.

[30] Li Li and Yujin Hu. "Nonlinear bending and free vibration analyses of nonlocal strain gradient beams made of functionally graded material". In: International Journal of Engineering Science 107 (2016), pp. 77-97.

[31] Li Li and Yujin Hu. "Wave propagation in fluid-conveying viscoelastic carbon nanotubes based on nonlocal strain gradient theory". In: Computational materials science 112 (2016), pp. 282-288.

[32] Li Li, Yujin Hu, and Xiaobai Li. "Longitudinal vibration of size-dependent rods via nonlocal strain gradient theory". In: International Journal of Mechanical Sciences 115 (2016), pp. 135-144.

[33] Li Li et al. "Size-dependent effects on critical flow velocity of fluid-conveying microtubes via nonlocal strain gradient theory". In: Microfluidics and nanofluidics 20.5 (2016), p. 76.

[34] Xiaobai Li et al. "Bending, buckling and vibration of axially functionally graded beams based on nonlocal strain gradient theory". In: Composite Structures 165 (2017), pp. 250-265.

[35] Xiaodong Li et al. "Mechanical characterization of micro/nanoscale structures for MEMS/NEMS applications using nanoindentation techniques". In: Ultramicroscopy 97.1-4 (2003), pp. 481-494.

[36] CW Lim, G Zhang, and JN Reddy. "A higher-order nonlocal elasticity and strain gradient theory and its applications in wave propagation". In: Journal of the Mechanics and Physics of Solids 78 (2015), pp. 298313.

[37] Hu Liu, Zheng Lv, and Han Wu. "Nonlinear free vibration of geometrically imperfect functionally graded sandwich nanobeams based on nonlocal strain gradient theory". In: Composite Structures 214 (2019), pp. 47-61.

[38] Rasheedat M Mahamood et al. "Functionally graded material: an overview". In: (2012).

[39] Fahimeh Mehralian, Yaghoub Tadi Beni, and Mehran Karimi Zeverdejani. "Calibration of nonlocal strain gradient shell model for buckling analysis of nanotubes using molecular dynamics simulations". In: Physica B: Condensed Matter 521 (2017), pp. 102-111.

[40] Salwa A Mohamed. "A fractional differential quadrature method for fractional differential equations and fractional eigenvalue problems". In: Mathematical Methods in the Applied Sciences (2020).

[41] Meisam Moory-Shirbani et al. "Experimental and mathematical analysis of a piezoelectrically actuated multilayered imperfect microbeam subjected to applied 
electric potential”. In: Composite Structures 184 (2018), pp. 950-960.

[42] Yves Moser and Martin AM Gijs. "Miniaturized flexible temperature sensor". In: Journal of Microelectromechanical Systems 16.6 (2007), pp. 1349-1354.

[43] KB Mustapha and ZW Zhong. "Free transverse vibration of an axially loaded non-prismatic single-walled carbon nanotube embedded in a two-parameter elastic medium". In: Computational Materials Science 50.2 (2010), pp. 742-751.

[44] Fehmi Najar et al. "Dynamics and global stability of beam-based electrostatic microactuators". In: Journal of Vibration and Control 16.5 (2010), pp. 721-748.

[45] Ali H Nayfeh and Walter Lacarbonara. "On the discretization of distributed-parameter systems with quadrati\$58] and cubic nonlinearities". In: Nonlinear Dynamics 13.3 (1997), pp. 203-220.

[46] Nikola Nešić et al. "Nonlinear superharmonic resonance analysis of a nonlocal beam on a fractional visco-Pasternak foundation". In: Proceedings of the Institution of Mechanical Engineers, Part C: Journal of Mechanical Engineering Science (2020), p. 09544062

[47] Ivan R Pavlović et al. "Dynamic behavior of two elastically connected nanobeams under a white noise process". In: Facta Universitatis, Series: Mechanical Engineering 18.2 (2020), pp. 219-227.

[48] John Peddieson, George R Buchanan, and Richard P McNitt. "Application of nonlocal continuum models to nanotechnology". In: International journal of engineering science 41.3-5 (2003), pp. 305-312.

[49] Igor Podlubny. Fractional differential equations: an introduction to fractional derivatives, fractional differential equations, to methods of their solution and some of their applications. Elsevier, 1998.

[50] SC Pradhan and JK Phadikar. "Nonlocal elasticity theory for vibration of nanoplates". In: Journal of Sound and Vibration 325.1-2 (2009), pp. 206-223.

[51] Venkatanarayanan Ramakrishnan and Brian F Feeny. "Resonances of a forced Mathieu equation with reference to wind turbine blades". In: Journal of vibration and acoustics 134.6 (2012).

[52] JN Reddy. "Nonlocal theories for bending, buckling and vibration of beams". In: International journal of engineering science 45.2-8 (2007), pp. 288-307.

[53] Shahab Saffari, Mohammad Hashemian, and Davood Toghraie. "Dynamic stability of functionally graded nanobeam based on nonlocal Timoshenko theory considering surface effects". In: Physica B: Condensed Matter 520 (2017), pp. 97-105.

[54] H Salehipour, AR Shahidi, and H Nahvi. "Modified nonlocal elasticity theory for functionally graded materials". In: International Journal of Engineering Science 90 (2015), pp. 44-57.
[55] Marina V Shitikova. "The fractional derivative expansion method in nonlinear dynamic analysis of structures". In: Nonlinear Dynamics 99.1 (2020), pp. 109_ 122.

[56] Mesut Şimşek. "Nonlinear free vibration of a functionally graded nanobeam using nonlocal strain gradient theory and a novel Hamiltonian approach". In: International Journal of Engineering Science 105 (2016), pp. 12-27.

[57] Pezhman Sourani et al. "A comparison of the Bolotin and incremental harmonic balance methods in the dynamic stability analysis of an Euler-Bernoulli nanobeam based on the nonlocal strain gradient theory and surface effects". In: Mechanics of Materials (2020), p. 103403. G Sales Teodoro, JA Tenreiro Machado, and E Capelas De Oliveira. "A review of definitions of fractional derivatives and other operators". In: Journal of Computational Physics 388 (2019), pp. 195-208.

[59] Necla Togun and Süleyman Murat Bağdatlı. "Nonlinear vibration of a nanobeam on a Pasternak elastic foundation based on non-local Euler-Bernoulli beam 22093632Beory". In: Mathematical and Computational Applications 21.1 (2016), p. 3.

[60] Hoang Lan Ton-That. "A new C0 third-order shear deformation theory for the nonlinear free vibration analysis of stiffened functionally graded plates". In: Facta Universitatis, Series: Mechanical Engineering 19.2 (2021), pp. 285-305.

[61] M Trabelssi, S El-Borgi, and MI Friswell. "A highorder FEM formulation for free and forced vibration analysis of a nonlocal nonlinear graded Timoshenko nanobeam based on the weak form quadrature element method". In: Archive of Applied Mechanics (2020), pp. 1-24.

[62] Gang-Feng Wang and Xi-Qiao Feng. "Effects of surface elasticity and residual surface tension on the natural frequency of microbeams". In: Applied physics letters 90.23 (2007), p. 231904.

[63] Jing Wang and Huoming Shen. "Nonlinear vibrations of axially moving simply supported viscoelastic nanobeams based on nonlocal strain gradient theory". In: Journal of Physics: Condensed Matter 31.48 (2019), p. 485403.

[64] Sheng Wang et al. "Applications of incremental harmonic balance method combined with equivalent piecewise linearization on vibrations of nonlinear stiffness systems”. In: Journal of Sound and Vibration 441 (2019), pp. 111-125.

[65] Zhong Lin Wang and Wenzhuo Wu. "Nanotechnologyenabled energy harvesting for self-powered micro-/nanosystems". In: Angewandte Chemie International Edition 51.47 (2012), pp. 11700-11721.

[66] Shao-Fang Wen et al. "Dynamical analysis of strongly nonlinear fractional-order Mathieu-Duffing equation”. 
In: Chaos: An Interdisciplinary Journal of Nonlinear Science 26.8 (2016), p. 084309.

[67] FACM Yang et al. "Couple stress based strain gradient theory for elasticity". In: International Journal of Solids and Structures 39.10 (2002), pp. 2731-2743.

[68] T Yokoyama. "Vibrations and transient responses of Timoshenko beams resting on elastic foundations". In: Ingenieur-Archiv 57.2 (1987), pp. 81-90.

[69] Davood Younesian et al. "Elastic and viscoelastic foundations: a review on linear and nonlinear vibration modeling and applications". In: Nonlinear Dynamics (2019), pp. 1-43.

[70] Paul M Zavracky et al. "Microswitches and microrelays with a view toward microwave applications". In: International Journal of RF and Microwave ComputerAided Engineering: Co-sponsored by the Center for Advanced Manufacturing and Packaging of Microwave, Optical, and Digital Electronics (CAMPmode) at the University of Colorado at Boulder 9.4 (1999), pp. 338347.

[71] Guoqi Zhang, Zhiqiang Wu, and Yajie Li. "Nonlinear Dynamic Analysis of Fractional Damped Viscoelastic Beams". In: Int. J. Struct. Stab. Dyn. 19 (11)(2019) 1950129 (2019).

[72] X Zhao et al. "Coupled thermoelastic nonlocal forced vibration of an axially moving micro/nano-beam". In: International Journal of Mechanical Sciences (2021), p. 106600.

[73] Yaxin Zhen and Lin Zhou. "Wave propagation in fluidconveying viscoelastic carbon nanotubes under longitudinal magnetic field with thermal and surface effect via nonlocal strain gradient theory". In: Modern Physics Letters B 31.08 (2017), p. 1750069.

[74] Yongtao Zhou et al. "Implicit-explicit time integration of nonlinear fractional differential equations". In: Applied Numerical Mathematics (2020).

\section{Appendix 1}

Elements of the Jacobi matrix $M=M_{1}+M_{2}^{\alpha}$, the corrective vector $R=R_{1}+R_{2}^{\alpha}$, and vector $V=V_{1}+V_{2}^{\alpha}$ are defined as:

$$
\begin{array}{r}
M_{1}=\frac{1}{2 \pi} \int_{0}^{2 \pi}\left[\Omega^{2} C^{T} \frac{d^{2} C}{d \bar{\tau}^{2}}+\omega_{0}^{2} C^{T} C+3 \theta q_{0}^{2} C^{T} C\right] d \bar{\tau} \\
M_{2}^{\alpha}=\frac{1}{T} \int_{0}^{T} C^{T}\left[\gamma \Omega^{\alpha} D_{\bar{\tau}}^{\alpha}(C)\right] d \bar{\tau} \\
R_{1}=-\frac{1}{2 \pi} \int_{0}^{2 \pi}\left[\left(\Omega^{2} C^{T} \frac{d^{2} C}{d \bar{\tau}^{2}}+\omega_{0}^{2} C^{T} C+\theta q_{0}^{2} C^{T} C\right) d \bar{\tau} A_{0}\right. \\
\left.+f_{0} C^{T}+f_{0} \cos \bar{\tau} C^{T}\right] d \bar{\tau}
\end{array}
$$

$R_{2}^{\alpha}=-\frac{1}{T} \int_{0}^{T} C^{T}\left[\gamma \Omega^{\alpha} D_{\bar{\tau}}^{\alpha}(C)\right] d \bar{\tau} A_{0}$

$V_{1}=\frac{1}{2 \pi} \int_{0}^{T}\left[2 \Omega_{0} C^{T} \frac{d^{2} C}{d \bar{\tau}^{2}}\right] d \bar{\tau} A_{0}$,

$V_{2}^{\alpha}=0$.

Within each incremental step, only a set of linear equations Eq.(58) has to be solved to obtain the data for the next stage. By applying the procedure established at $[66,46]$ elements of the matrix $M_{2}^{\alpha}$, and vectors $R_{2}^{\alpha}$ and $V_{2}^{\alpha}$ can be expressed as

$$
M_{2}^{\alpha}=\left[\begin{array}{ll}
{\left[M_{11}\right]^{\alpha}} & {\left[M_{12}\right]^{\alpha}} \\
{\left[M_{21}\right]^{\alpha}} & {\left[M_{22}\right]^{\alpha}}
\end{array}\right], \quad R_{2}^{\alpha}=\left[\begin{array}{c}
R_{10}^{\alpha} \\
R_{1}^{\alpha} \\
R_{2}^{\alpha}
\end{array}\right], \quad V_{2}^{\alpha}=\left[\begin{array}{c}
V_{10}^{\alpha} \\
V_{1}^{\alpha} \\
V_{2}^{\alpha}
\end{array}\right] .
$$

Elements of matrix $M_{2}^{\alpha}$, and vectors $R_{2}^{\alpha}$ and $V_{2}^{\alpha}$ from Eq.(73) are:

$$
\begin{gathered}
{\left[M_{11}\right]_{i j}^{\alpha}=\delta_{i j} \gamma \Omega^{\alpha} \frac{i^{\alpha}}{2} \cos \left(\frac{\alpha \pi}{2}\right),} \\
i=0,1,2, \ldots, N, \quad j=0,1,2, \ldots, N, \\
{\left[M_{12}\right]_{i j}^{\alpha}=\delta_{i j} \gamma \Omega^{\alpha} \frac{i^{\alpha}}{2} \sin \left(\frac{\alpha \pi}{2}\right),} \\
i=0,1,2, \ldots, N, \quad j=1,2, \ldots, N, \\
{\left[M_{21}\right]_{i j}^{\alpha}=-\delta_{i j} \gamma \Omega^{\alpha} \frac{i^{\alpha}}{2} \sin \left(\frac{\alpha \pi}{2}\right),} \\
i=1,2, \ldots, N, \quad j=0,1,2, \ldots, N, \\
{\left[M_{22}\right]_{i j}^{\alpha}=\delta_{i j} \gamma \Omega^{\alpha} \frac{i^{\alpha}}{2} \cos \left(\frac{\alpha \pi}{2}\right),} \\
i=1,2, \ldots, N, \quad j=1,2, \ldots, N,
\end{gathered}
$$

$R_{10}^{\alpha}=0$,

$$
\begin{array}{r}
R_{1 i}^{\alpha}=-\gamma \Omega^{\alpha}\left[a_{i} \frac{i^{\alpha}}{2} \cos \left(\frac{\alpha \pi}{2}\right)+b_{i} \frac{i^{\alpha}}{2} \sin \left(\frac{\alpha \pi}{2}\right)\right], \\
i=1,2, \ldots, N, \\
R_{2 i}^{\alpha}=-\gamma \Omega^{\alpha}\left[a_{i} \frac{i^{\alpha}}{2} \sin \left(\frac{\alpha \pi}{2}\right)+b_{i} \frac{i^{\alpha}}{2} \cos \left(\frac{\alpha \pi}{2}\right)\right], \\
i=1,2, \ldots, N,
\end{array}
$$

$$
\begin{gathered}
V_{10}^{\alpha}=0, \\
V_{1 i}^{\alpha}=\gamma \alpha \Omega^{\alpha-1}\left[a_{i} \frac{i^{\alpha}}{2} \cos \left(\frac{\alpha \pi}{2}\right)+b_{i} \frac{i^{\alpha}}{2} \sin \left(\frac{\alpha \pi}{2}\right)\right], \\
i=1,2, \ldots, N, \\
V_{2 i}^{\alpha}=\gamma \alpha \Omega^{\alpha-1}\left[a_{i} \frac{i^{\alpha}}{2} \sin \left(\frac{\alpha \pi}{2}\right)+b_{i} \frac{i^{\alpha}}{2} \cos \left(\frac{\alpha \pi}{2}\right)\right], \\
i=1,2, \ldots, N,
\end{gathered}
$$

where $\delta_{i j}$ is Kronecker delta. 
Appendix 2

Multiple scales method

Multiple scales is the analytical perturbation method for constructing approximate solutions of nonlinear differential equations. This method is well established in the literature but it is only valid for small nonlinearities and damping. Therefore, we will use it here only for validation purposes. Eq.(46) is well known as the forced Duffing fractional-order differential equation, which can be expressed in terms of small scale parameter $\varepsilon$ as in Eq.(77). Let assume for simplicity $f_{0}=0, f=f_{1}$.

$\ddot{q}+\varepsilon \bar{\gamma} D_{\tau}^{\alpha} q+\omega_{0}^{2} q+\varepsilon \bar{\theta} q^{3}=f \cos \Omega \tau$.

Here, we introduce new parameters as $\gamma=\varepsilon \bar{\gamma}$ and $\theta=\varepsilon \bar{\theta}$. The small bookkeeping parameter $\varepsilon$ is put in front of the fractional and nonlinear terms to have weak damping and weak nonlinearity. Please note that the forcing term in Eq.(77) is of the order one (also known as hard forcing) which will help us to study secondary resonances in the system by using the perturbation analysis of the first order. Forcing of order $\varepsilon$ would indicate a primary resonance that is the same as in the Duffing equation [51].

Using the multiple scales method, we will seek the solution of Eq.(77) in the following form:

$q\left(T_{0}, T_{1}, \varepsilon\right)=q_{0}\left(T_{0}, T_{1}\right)+\varepsilon q_{1}\left(T_{0}, T_{1}\right)+\cdots$.

Here, $T_{0}=\tau$ is the fast time scale and $T_{1}=\varepsilon \tau$ is the slow time scale. We will analyze the system for superharmonic resonance conditions. Firstly, let us define the time derivatives as

$\frac{d}{d \tau}=D_{0}+\varepsilon D_{1}+O\left(\varepsilon^{2}\right)$,

$\frac{d^{2}}{d \tau^{2}}=D_{0}^{2}+2 \varepsilon D_{0} D_{1}+O\left(\varepsilon^{2}\right)$,

$D^{\alpha}=D_{0+}^{\alpha}-\varepsilon \alpha D_{0+}^{\alpha-1} D_{1}+\cdots$,

where $D_{n}=\frac{\partial}{\partial T_{n}},(n=0,1,2, \ldots)$ and $D_{n+}^{\alpha-n}=\frac{\partial^{\alpha-n}}{\partial T_{n+}^{\alpha-n}},(n=$ $0,1,2, \ldots)$ are classical and Riemann-Liouville's fractional derivative for new time scales [55]. For the fractional derivative of the exponential function [55], restricted to the first and second-order approximations, the following relationship will be used:

$D_{0+}^{\alpha} \exp ^{i \omega \tau}=(i \omega)^{\alpha} \exp ^{i \omega \tau}$

where $i$ is the imaginary unit. Substituting Eqs. (78), (79), (80), (81) into Eq.(77) and then extracting coefficients of $\varepsilon^{0}$ and $\varepsilon^{1}$ we obtain the following equations

$\varepsilon^{0}: D_{0}^{2} q_{0}+\omega_{0}^{2} q_{0}=f \cos \Omega \tau$, $\varepsilon^{1}: D_{0}^{2} q_{1}+\omega_{0}^{2} q_{1}=-2 D_{0} D_{1} q_{0}-\bar{\gamma} D_{0+}^{\alpha} q_{0}-\bar{\theta} q_{0}^{3}$.

The solution of Eq.(83) is sought in the form

$q_{0}=A\left(T_{1}\right) e^{i \omega_{0} T_{0}}+\Lambda e^{i \Omega T_{1}}+\bar{A}\left(T_{1}\right) e^{-i \omega_{0} T_{0}}+\Lambda e^{-i \Omega T_{1}}$,

where $A$ is a complex function in terms of slow time scale, and $\Lambda$ is defined as

$\Lambda=\frac{f}{2\left(\omega_{0}^{2}-\Omega^{2}\right)}$.

Superharmonic resonance $3 \Omega \approx \omega_{0}$

Since we have only cubic nonlinearity in Eq.(77), we will consider the case when $3 \Omega=\omega_{0}+\varepsilon \sigma$, where $\sigma$ is the detuning parameter. By substituting $q_{0}$ from Eq.(85) into Eq.(84) and removing the secular terms that grow in time unbounded, i.e the coefficients of $e^{i \omega_{0} T_{0}}$, we obtain the corresponding solvability conditions as

$-2 i \omega_{0} A^{\prime}-\bar{\gamma} A\left(i \omega_{0}\right)^{\alpha}-\bar{\theta}\left(3 A^{2} \bar{A}+6 A \Lambda^{2}+\Lambda^{3} e^{i \sigma T_{1}}\right)=0$,

where $A^{\prime}=D_{1} A$. Then, we use the polar form $A=\frac{1}{2} a e^{i \varphi}$, where the real valued functions $a$ and $\varphi$ are the amplitude and phase lag of time response, respectively. By substituting $A$ in Eq.(87) and separation of real and imaginary part we obtain

$\omega_{0} a \varphi^{\prime}-\frac{1}{2} \bar{\gamma} a \omega_{0}^{\alpha} \cos \frac{\alpha \pi}{2}-\frac{3}{8} \bar{\theta} a^{3}-3 \bar{\theta} a \Lambda^{2}-\bar{\theta} \Lambda^{3} \cos \zeta=0$,

$\omega_{0} a^{\prime}+\frac{1}{2} \bar{\gamma} a \omega_{0}^{\alpha} \sin \frac{\alpha \pi}{2}+\bar{\theta} \Lambda^{3} \sin \zeta=0$,

with $\zeta=\sigma T_{1}-\varphi$ denoting the new phase angle. Then, we utilize steady-state conditions $a^{\prime}=0, \zeta^{\prime}=0$ in Eq.(88) and Eq.(89), which leads to the relationship between the response amplitude and the detuning parameter in the following form

$\frac{\bar{\theta} \Lambda^{3}}{\omega_{0} a} \cos \zeta=\sigma-\frac{1}{2} \bar{\gamma} \omega_{0}^{\alpha-1} \cos \frac{\alpha \pi}{2}-\frac{3}{8} \frac{\bar{\theta} a^{2}}{\omega_{0}}-3 \frac{\bar{\theta} \Lambda^{2}}{\omega_{0}}$

$\frac{\bar{\theta} \Lambda^{3}}{\omega_{0} a} \sin \zeta=-\frac{1}{2} \bar{\gamma} \omega_{0}^{\alpha-1} \sin \frac{\alpha \pi}{2}$.

After simple algebra transformations over Eq.(90) and Eq.(91) following polynomial equation can be obtained

$\sigma^{2}-2 \sigma K+M=0$

with $K$ and $M$ given as

$K=\frac{1}{2} \bar{\gamma} \omega_{0}^{\alpha-1} \cos \frac{\alpha \pi}{2}+\frac{3}{8} \frac{\bar{\theta} a^{2}}{\omega_{0}}-3 \frac{\bar{\theta} \Lambda^{2}}{\omega_{0}}$,

$M=K^{2}+\left(\frac{1}{2} \bar{\gamma} \omega_{0}^{\alpha-1} \sin \frac{\alpha \pi}{2}\right)^{2}$, 
from where the relationship for amplitude-frequency curves can be obtained as

$\sigma_{1,2}=K \pm \sqrt{K^{2}-M}$.

One can notice that all the parameters contribute to the appearance of the superharmonic resonance of order $1 / 3$ i.e. we have interaction of terms of fractional-order, nonlinear, and external excitation.

\section{Appendix 3}

Newmark method

We use Grunwald-Letnikov representation of fractional derivative and apply the Newmark-Beta method for numerical integration. We use two different meshes, coarse mesh for time integration and fine mesh for fractional derivative approximation. Grunwald-Letnikov representation of a fractional derivative of a function $q(\bar{\tau})$ at a point of time $\bar{\tau}$ is

${ }_{G L} D_{0, \bar{\tau}}^{\alpha} q(\bar{\tau})=\lim _{h \rightarrow 0} h^{-\alpha} \sum_{k=0}^{n} G L_{k} q(\bar{\tau}-k h)$,

where

$G L_{k}=(-1)^{k}\left(\begin{array}{l}\alpha \\ k\end{array}\right)$

Grunwald-Letnikov coefficients can also be represented in recursive form as

$G L_{k=0}=1, \quad G L_{k}=\frac{k-\alpha-1}{k} G L_{k-1}$.

Let us define

$\frac{\Delta \bar{\tau}}{h}=p=5 \div 20$.

where $\Delta \bar{\tau}$ is time step for coarse mesh, and $h$ is time step for fine mesh.

Representation of fractional derivative given by Eq.(96) in fine mesh is

$q_{i}^{(\alpha)}=h^{-\alpha}\left[\begin{array}{lll}G L_{0} & G L_{1} \cdots & G L_{k j p}\end{array}\right]\left[\begin{array}{c}q_{i} \\ q_{i-1} \\ \vdots \\ q_{i-p} \\ q_{i-p-1} \\ \vdots \\ q_{i-2 p} \\ q_{i-2 p-1} \\ \vdots \\ q_{k j p}\end{array}\right]$,

where: $p$ is the number of past terms of length $\mathrm{h}$ in a time integration step of length $\Delta \bar{\tau}$,

$j$ are previous time steps of length $\Delta \bar{\tau}$ that can be approximated accurately by a backward Taylor expansion using the displacement, velocity, and acceleration at a certain time step $i$,

$k$ represents overall chunks of $j$ time steps that must be taken into consideration to accurately approximate the fractional derivative at a given point.

Taylor backward expansion for the last $j p$ time steps can be represented as in Eq.(101),

$$
\begin{aligned}
q_{i-1} & =q_{i}-h \dot{q}_{i}+\frac{h^{2}}{2} \ddot{q}_{i}+O\left(h^{3}\right), \\
q_{i-2} & =q_{i}-2 h \dot{q}_{i}+\frac{4 h^{2}}{2} \ddot{q}_{i}+O\left(h^{3}\right), \\
q_{i-3} & =q_{i}-3 h \dot{q}_{i}+\frac{9 h^{2}}{2} \ddot{q}_{i}+O\left(h^{3}\right), \\
\vdots & \\
q_{i-j p} & =q_{i}-j p h \dot{q}_{i}+\frac{j^{2} p^{2} h^{2}}{2} \ddot{q}_{i}+O\left(h^{3}\right),
\end{aligned}
$$

where $q_{i}, \dot{q}_{i}$ and $\ddot{q}_{i}$ are displacement, velocity and acceleration, respectfully, at time step $i$.

Lets neglect higher order terms. Eq.(101) can be written in the matrix form as Eq.(102),

$$
\begin{aligned}
{\left[\begin{array}{c}
q_{i} \\
q_{i-1} \\
q_{i-2} \\
q_{i-3} \\
\vdots \\
q_{i-(j p-1)}
\end{array}\right]=\left[\begin{array}{ccc}
1 & 0 & 0 \\
1 & -h & \frac{h^{2}}{2} \\
1 & -2 h & \frac{4 h^{2}}{2} \\
1 & -3 h & \frac{9 h^{2}}{2} \\
\vdots & \vdots & \vdots \\
1-(j p-1) h \frac{(j p-1)^{2} h^{2}}{2}
\end{array}\right]\left[\begin{array}{c}
q_{i} \\
\dot{q}_{i} \\
\ddot{q}_{i}
\end{array}\right] } \\
=\left[H_{0}\right]\left[\begin{array}{c}
q_{i} \\
\dot{q}_{i} \\
\ddot{q}_{i}
\end{array}\right] .
\end{aligned}
$$

By analogy, the displacements from the step $i-j p$ to the $i-$ $(2 j p-1)$ in matrix form in terms of displacements, velocity and acceleration of the $i-j p$ is given by the Eq.(103),

$$
\begin{aligned}
{\left[\begin{array}{c}
q_{i-j p} \\
q_{i-j p-1} \\
q_{i-j p-2} \\
q_{i-j p-3} \\
\vdots \\
q_{i-(2 j p-1)}
\end{array}\right]=\left[\begin{array}{ccc}
1 & 0 & 0 \\
1 & -h & \frac{h^{2}}{2} \\
1 & -2 h & \frac{4 h^{2}}{2} \\
1 & -3 h & \frac{9 h^{2}}{2} \\
\vdots & \vdots & \vdots \\
1-(j p-1) h & \frac{(j p-1)^{2} h^{2}}{2}
\end{array}\right]\left[\begin{array}{c}
q_{i-j} \\
\dot{q}_{i-j} \\
\ddot{q}_{i-j}
\end{array}\right] } \\
=[H]\left[\begin{array}{c}
q_{i-j} \\
\dot{q}_{i-j} \\
\ddot{q}_{i-j}
\end{array}\right] .
\end{aligned}
$$


Here could jerk also be included, but we didn't do this. Since we omitted jerk, $[H]=\left[H_{0}\right]$. Substituting Eq.(101), Eq.(102) and Eq.(103) in Eq.(100) we obtain following expressions:

$$
\begin{aligned}
& q_{i}^{(\alpha)}=h^{-\alpha}\left[G L_{0} G L_{1} \cdots G L_{j p-1}\right]\left[H_{0}\right]\left[\begin{array}{c}
q_{i} \\
\dot{q}_{i} \\
\ddot{q}_{i}
\end{array}\right] \\
& +h^{-\alpha}\left[G L_{j p} G L_{j p+1} \cdots G L_{2 j p-1}\right][H]\left[\begin{array}{c}
q_{i-j} \\
\dot{q}_{i-j} \\
\ddot{q}_{i-j}
\end{array}\right]+\cdots \\
& +h^{-\alpha}\left[G L_{(k-1) j p} \cdots G L_{k j p-1}\right][H]\left[\begin{array}{l}
q_{i-(k-1) j} \\
\dot{q}_{i-(k-1) j} \\
\ddot{q}_{i-(k-1) j}
\end{array}\right],
\end{aligned}
$$

$$
\begin{aligned}
q_{i}^{(\alpha)}= & {\left[\begin{array}{lll}
D_{01} & D_{02} & D_{03}
\end{array}\right]\left[\begin{array}{c}
q_{i} \\
\dot{q}_{i} \\
\ddot{q}_{i}
\end{array}\right]+\left[\begin{array}{lll}
D_{11} & D_{12} & D_{13}
\end{array}\right]\left[\begin{array}{c}
q_{i-j} \\
\dot{q}_{i-j} \\
\ddot{q}_{i-j}
\end{array}\right] } \\
& +\cdots+\left[\begin{array}{lll}
D_{(k-1) 1} & D_{(k-1) 2} & D_{(k-1) 3}
\end{array}\right]\left[\begin{array}{l}
q_{i-(k-1) j} \\
\dot{q}_{i-(k-1) j} \\
\ddot{q}_{i-(k-1) j}
\end{array}\right],
\end{aligned}
$$

$$
\begin{aligned}
\Delta q_{i}^{(\alpha)}= & {\left[\begin{array}{lll}
D_{01} & D_{02} & D_{03}
\end{array}\right]\left[\begin{array}{c}
\Delta q_{i} \\
\Delta \dot{q}_{i} \\
\Delta \ddot{q}_{i}
\end{array}\right]+\left[\begin{array}{lll}
D_{11} & D_{12} & D_{13}
\end{array}\right]\left[\begin{array}{c}
\Delta q_{i-j} \\
\Delta \dot{q}_{i-j} \\
\Delta \ddot{q}_{i-j}
\end{array}\right] } \\
& +\cdots+\left[\begin{array}{lll}
D_{(k-1) 1} & D_{(k-1) 2} & D_{(k-1) 3}
\end{array}\right]\left[\begin{array}{c}
\Delta q_{i-(k-1) j} \\
\Delta \dot{q}_{i-(k-1) j} \\
\Delta \ddot{q}_{i-(k-1) j}
\end{array}\right] .
\end{aligned}
$$

Lets consider equation of motion Eq.(48) in two consecutive time instants:

$$
\begin{aligned}
& \Omega^{2} \Delta \ddot{q}_{i}+\omega_{0}^{2} \Delta q_{i}+\theta\left(\Delta q_{i}\right)^{3} \\
& +\gamma \Omega^{\alpha}\left({ }_{G L} D_{0, \bar{\tau}_{i}}^{\alpha} q_{i}-{ }_{G L} D_{0, \bar{\tau}_{i-1}}^{\alpha} q_{i-1}\right)=f_{0}+\Delta f_{i},
\end{aligned}
$$

where

$\Delta f_{i}=f_{1} \cos \bar{\tau}_{i}$

By substituting Eq.(106) in Eq.(107) we obtain

$$
\begin{aligned}
& \left(\Omega^{2}+\gamma \Omega^{\alpha} D_{03}\right) \Delta \ddot{q}_{i}+\gamma \Omega^{\alpha} D_{02} \Delta \dot{q}_{i}+\left(\omega_{0}^{2}+\gamma \Omega^{\alpha} D_{01}\right) \Delta q_{i} \\
& \quad+\theta\left(\Delta q_{i}\right)^{3}=f_{0}+\Delta f_{i}-\Delta f_{\text {correction }}
\end{aligned}
$$

where

$$
\begin{gathered}
\Delta f_{\text {correction }}=\gamma \Omega^{\alpha}\left[\begin{array}{lll}
D_{11} & D_{12} & D_{13}
\end{array}\right]\left[\begin{array}{l}
\Delta q_{i-j} \\
\Delta \dot{q}_{i-j} \\
\Delta \ddot{q}_{i-j}
\end{array}\right]+\cdots+ \\
\gamma \Omega^{\alpha}\left[\begin{array}{lll}
D_{(k-1) 1} & D_{(k-1) 2} & D_{(k-1) 3}
\end{array}\right]\left[\begin{array}{l}
\Delta q_{i-(k-1) j} \\
\Delta \dot{q}_{i-(k-1) j} \\
\Delta \ddot{q}_{i-(k-1) j}
\end{array}\right] .
\end{gathered}
$$

Note that in case of $\Delta f_{i}=$ const, Eq.(109) can be solved using Runge-Kutta method (function ode45 in Matlab). If this is not the case, Eq.(109) can be solved using NewmarkBeta method.

For validation of the IHB solution, the Newmark-Beta method for nonlinear systems is used and implemented according to the procedure presented in $[6,12]$. 